\title{
DELTA METHODS IN ENVELOPING ALGEBRAS OF LIE SUPERALGEBRAS
}

\author{
JEFFREY BERGEN AND D. S. PASSMAN
}

\begin{abstract}
Let $L$ be a Lie superalgebra over a field $K$ of characteristic $\neq 2$. We define

$$
\Delta(L)=\left\{l \in L \mid \operatorname{dim}_{K}[L, l]<\infty\right\} .
$$

Then $\Delta(L)$ is a Lie ideal of $L$ and is restricted if $L$ is restricted. $\Delta(L)$ is the Lie superalgebra analog of the Lie delta ideal, used by the authors in the study of enveloping rings, and also of the finite conjugate center of a group, used in the study of group algebras and crossed products.

In this paper we examine $U(L)$, where depending upon char $K, U(L)$ denotes either the enveloping algebra or the restricted enveloping algebra of $L$. We show that $\Delta(L)$ controls certain properties of $U(L)$. Specifically, we consider semiprimeness, primeness, almost constants, almost centralizers, central closures, and the Artinian condition.
\end{abstract}

\section{INTRODUCTION}

Let $L=L_{0} \oplus L_{1}$ be a Lie superalgebra over a field $K$ of characteristic $\neq 2$. If char $K=0$, we let $U(L)$ denote its enveloping algebra and if char $K=$ $p>2$, we will assume that $L$ is restricted and $U(L)$ will denote its restricted enveloping algebra. In the study of group algebras and enveloping algebras of ordinary Lie algebras, many questions have been solved using $\Delta$-methods. It is reasonable to try to find similar techniques in the Lie superalgebra context. To this end, we consider

$$
\Delta(L)=\left\{l \in L \mid \operatorname{dim}_{K}[L, l]<\infty\right\},
$$

which is a (restricted) Lie ideal of $L$.

The goal is to reduce questions about $U(L)$ to analogous ones for $U(\Delta(L))$. We begin in $\S 1$ with the definitions and terminology for Lie superalgebras and their enveloping algebras. In $\S 2$, we examine the basic properties of $\Delta(L)$. In $\S 3$, we discuss derivation identities, namely expressions of the form

$$
\alpha_{1}^{\delta} \beta_{1}+\alpha_{2}^{\delta} \beta_{2}+\cdots+\alpha_{v}^{\delta} \beta_{v}=0 \quad \text { for all } \delta \in \partial^{\infty}(L),
$$

where $\partial^{\infty}(L)$ is the set of all multiple superderivations induced, via the ad map, by all elements of $L_{0}$ and $L_{1}$.

The main result is

Received by the editors February 14, 1990 and, in revised form, September 10, 1990.

1980 Mathematics Subject Classification (1985 Revision). Primary 17B35, 17A70, 17B65.

The second author's research was supported, in part by NSF Grant No. DMS 8900405 . 
Theorem. Suppose $\alpha_{i}, \beta_{i} \in U(L)$ such that

$$
\alpha_{1}^{\delta} \beta_{1}+\alpha_{2}^{\delta} \beta_{2}+\cdots+\alpha_{v}^{\delta} \beta_{v}=0 \quad \text { for all } \delta \in \partial^{\infty}(L) .
$$

If some $\alpha_{i}$ is not zero, then $\beta_{1}, \beta_{2}, \ldots, \beta_{v}$ are left dependent over $U(\Delta(L))$.

In $\S 4$, we introduce linear identities, that is equations of the form

$$
\alpha_{1} s \beta_{1}+\alpha_{2} s \beta_{2}+\cdots+\alpha_{v} s \beta_{v}=0 \text { for all } s \in U(L) \text {. }
$$

From a ring theoretic point of view, linear identities are more important than derivation identities as they arise in numerous ring theoretic situations. For enveloping algebras of ordinary Lie algebras, linear identities always yield corresponding derivation identities [BP]. However, for superalgebras this is not necessarily the case. Thus our results for linear identities are not quite as sharp as those for derivation identities.

We apply the results of derivation and linear identities in $\S 5$. We show in Corollary 5.6 that $U(L)$ is graded prime if and only if $U(\Delta(L))$ is graded $L$ prime and that $U(L)$ is semiprime if and only if $U(\Delta(L))$ is $L$-semiprime. When $\Delta(L)=0$, we show that $U(L)$ is prime and that if $Q$ is its symmetric Martindale quotient ring, then both the center of $Q$ and the almost constants in $Q$ under the action of $L$ are equal to the ground field $K$. We also show that $U(L)$ is Artinian if and only if it is finite dimensional.

\section{DEFINITIONS AND TERMINOLOGY}

Let $R$ be an associative algebra over a field $K$ of characteristic $\neq 2$. If $\sigma$ is a $K$-linear automorphism of $R$ such that $\sigma^{2}=1$, then $R$ is graded by $\mathbb{Z}_{2}$. More precisely, we can write $R=R_{0} \oplus R_{1}$, where $R_{0}=\left\{r \in R \mid r^{\sigma}=r\right\}$ and $R_{1}=\left\{r \in R \mid r^{\sigma}=-r\right\}$. Both $R_{0}$ and $R_{1}$ are $K$-subspaces of $R$ and $R_{a} R_{b} \subseteq R_{a+b}$, where $a+b$ is computed modulo 2 .

We can define a $K$-linear superbracket [, ] on $R$ by

(1) $[x, y]=x y-(-1)^{a b} y x$, for all $x \in R_{a}, y \in R_{b}$.

It follows that [, ] satisfies

(2) $[y, x]=-(-1)^{a b}[x, y]$, for all $x \in R_{a}, y \in R_{b}$,

(3) $(-1)^{a c}[x,[y, z]]+(-1)^{a b}[y,[z, x]]+(-1)^{b c}[z,[x, y]]=0$, for all $x \in$ $R_{a}, y \in R_{b}, z \in R_{c}$

(4) $\left[x^{\sigma}, y^{\sigma}\right]=[x, y]^{\sigma}$, for all $x, y \in R$;

(5) $[x, y z]=[x, y] z+(-1)^{a b} y[x, z]$, for all $x \in R_{a}, y \in R_{b}, z \in R_{c}$.

Property (2) is called graded skew-symmetry, property (3) is called the graded Jacobi identity, and property (5) is known as the super derivation property.

The proof of (2) is straightforward and the proofs of (3) and (5) follow easily from the fact that $\left[R_{a}, R_{b}\right] \subseteq R_{a+b}$. Since $\sigma$ is a ring homomorphism which preserves $R_{0}$ and $R_{1}$, (4) holds for all elements of $R_{0}$ and $R_{1}$ and therefore, by linearity, for all elements of $R$.

Some basic properties are

Lemma 1.1. Let $x \in R_{a}$.

(i) If $z=z_{0}+z_{1}$, with $z_{i} \in R_{i}$, then $[x, z]=0$ if and only if $\left[x, z_{0}\right]=0$ and $\left[x, z_{1}\right]=0$.

(ii) If $y, z \in R$ and $[x, z]=0$, then $[x, y z]=[x, y] z$.

(iii) $\{r \in R \mid[x, r]=0\}$ is a $\sigma$-stable subring of $R$.

Proof. (i) This is clear, since $\left[x, z_{i}\right] \in R_{a+i}$, for $i=0,1$. 
(ii) By linearity and by (i), we may assume that $y \in R_{b}$ and $z \in R_{c}$. The result then follows by applying property (5).

(iii) Let $A=\{r \in R \mid[x, r]=0\}$; by (ii), $A$ is closed under multiplication and $A$ is clearly closed under addition. If $r=r_{0}+r_{1}$, with $r_{i} \in R_{i}$, then $\left[x, r^{\sigma}\right]=\left[x, r_{0}\right]-\left[x, r_{1}\right]$, thus $A$ is $\sigma$-stable by $(\mathrm{i})$.

We note that a subspace $I \subseteq R$ is $\sigma$-stable if and only if $I=\left(I \cap R_{0}\right) \oplus(I \cap$ $\left.R_{1}\right)=I_{0} \oplus I_{1}$. Indeed, if $I=I_{0} \oplus I_{1}$, then $I$ is $\sigma$-stable as each $I_{a}$ is $\sigma$-stable and conversely, if $I$ is $\sigma$-stable then it certainly admits such a decomposition. The elements of $I_{0}$ and $I_{1}$ are referred to as the homogeneous elements of $I$. We will also often use the term graded in place of $\sigma$-stable.

If $x \in R$ then those $r \in R$ such that $[x, r]=0$ will be referred to as the constants for $x$. Note that if $x \in R_{a}$ and if $z$ is a constant for $x$, then the analog of (ii) fails when computing $[x, z y]$ since a + or - sign occurs when $z$ is homogeneous, hence we cannot apply linearity.

Now suppose $L$ is a $\sigma$-stable subspace of $R$ such that $[L, L] \subseteq L$. Then [ , ] on $L$ is $K$-linear and satisfies (2) and (3). This leads us to the abstract definition of a Lie superalgebra.

Definition. Suppose $L$ is a vector space over a field $K$ of characteristic $\neq 2$ and suppose $\sigma$ is a $K$-linear transformation of $L$ such that $\sigma^{2}=1 . L$ then has a decomposition $L=L_{0} \oplus L_{1}$, where $L_{0}=\left\{\left.l \in L\right|^{\sigma}=l\right\}$ and $L_{1}=\left\{l \in L \mid l^{\sigma}=-l\right\}$. We say that $L$ is a Lie superalgebra if there is a $K$-linear map [ , ] satisfying

(2) $[y, x]=-(-1)^{a b}[x, y]$, and

(3) $(-1)^{a c}[x,[y, z]]+(-1)^{a b}[y,[z, x]]+(-1)^{b c}[z,[x, y]]=0$, for all $x \in$ $R_{a}, y \in R_{b}$, and $z \in R_{c}$.

If $L$ is a Lie superalgebra, then the Poincaré-Birkhoff-Witt Theorem guarantees that there exists a unique largest $K$-algebra $U(L) \supseteq L$ such that $U(L)$ is generated by $L$ with the relations $x y-(-1)^{a b} y x=[x, y]$, for all $x \in L_{a}$ and $y \in L_{b}$. More precisely (see [B] or [S]), we have

Poincaré-Birkhoff-Witt Theorem. Let $B$ be a totally ordered basis for $L$ consisting of homogeneous elements. Then $U(L)$ has as a $K$-basis all ordered monomials $b_{1}^{\beta_{1}} b_{2}^{\beta_{2}} \cdots b_{n}^{\beta_{n}}$, such that $b_{i} \in B, b_{1}<b_{2}<\cdots<b_{n}, \beta_{i}$ are nonnegative integers, and $\beta_{i} \leq 1$ whenever $b_{i} \in L_{1}$.

If char $K=p>2$, there is an additional structure one can add.

Definition. $L$ is a restricted Lie superalgebra if it is a Lie superalgebra with a $p$ th power map $L_{0} \rightarrow L_{0}$, denoted by ${ }^{[p]}$, satisfying

(6) $(k x)^{[p]}=k^{p} x^{[p]}$, for all $k \in K$ and $x \in L_{0}$;

(7) $\left[x^{[p]}, y\right]=(\operatorname{ad} x)^{p}(y)$, for all $x \in L_{0}$ and $y \in L$;

(8) $(x+y)^{[p]}=x^{[p]}+y^{[p]}+\sum_{i=1}^{p-1} s_{i}(x, y)$, for all $x, y \in L_{0}$;

where $(\operatorname{ad} x)(y)=[x, y]$ and $i s_{i}(x, y)$ is the coefficient of $\lambda^{i-1}$ in $(\operatorname{ad}(\lambda x+y))^{p-1}(x)$.

If $L$ is a restricted Lie superalgebra, then there exists a unique largest $K$ algebra $U(L) \supseteq L$ such that $U(L)$ is generated by $L$ with relations $x y-$ $(-1)^{a b} y x=[x, y]$, for all $x \in L_{a}$ and $y \in L_{b}$, and $x^{p}=x^{[p]}$, for all $x \in L_{0}$. The obvious analog of Jacobson's Theorem on restricted enveloping algebras asserts that $U(L)$ exists (see [P]). Indeed we have 
Jacobson's Theorem. Let $L$ be a restricted Lie superalgebra in char $p>2$ and let $B$ be a totally ordered basis for $L$ consisting of homogeneous elements. Then $U(L)$ has as a $K$-basis all ordered monomials $b_{1}^{\beta_{1}} b_{2}^{\beta_{2}} \cdots b_{n}^{\beta_{n}}$, such that $b_{i} \in B$, $b_{1}<b_{2}<\cdots<b_{n}$, and such that $0 \leq \beta_{i}<p$ whenever $b_{i} \in L_{0}$, and $0 \leq \beta_{i} \leq 1$ whenever $b_{i} \in L_{1}$.

Regardless of the characteristic of $K$, we will refer to $U(L)$ as the enveloping algebra of $L$. However, the meaning of $U(L)$ depends upon the characteristic of $K$. If char $K=0$ then $U(L)$ is the algebra with $K$-basis described by the Poincaré-Birkhoff-Witt Theorem, whereas if char $K=p>2$ then we will assume that $L$ is restricted and $U(L)$ has a $K$-basis described by Jacobson's Theorem.

Those monomials which occur in the Poincaré-Birkhoff-Witt Theorem or in Jacobson's Theorem are said to be straightened.

If $X$ is a subset of $L$, we let $X^{n}$ denote the $K$-linear span of all products of the form $x_{1} x_{2} \cdots x_{k}$, where $x_{i} \in X$ and $0 \leq k \leq n$. We note that $U(L)=$ $\bigcup_{n=0}^{\infty} L^{n}$ and if $X^{\sigma}=X$, then $X^{1} L^{1}=L^{1} X^{1}$. In addition, we set $X^{\infty}=$
$\bigcup_{n=0}^{\infty} X^{n}$.

If $0 \neq \alpha \in U(L)$, then the degree of $\alpha$ is the minimal $m$ with $\alpha \in L^{m}$. From the Poincaré-Birkhoff-Witt Theorem, it follows that if $b_{1}^{\beta_{1}} b_{2}^{\beta_{2}} \cdots b_{n}^{\beta_{n}}$ is a straightened monomial, then its degree is $\beta_{1}+\beta_{2}+\cdots+\beta_{n}$. Furthermore, if $\alpha$ is given by

$$
\alpha=\sum k_{\beta} b_{1}^{\beta_{1}} b_{2}^{\beta_{2}} \cdots b_{n}^{\beta_{n}},
$$

then the degree of $\alpha, \operatorname{deg} \alpha$, is $\max \left\{\beta_{1}+\beta_{2}+\cdots+\beta_{n} \mid k_{\beta} \neq 0\right\}$.

Finally, since $\sigma$ is an automorphism of $L$ of order 2, it extends to an automorphism of order 2 of $U(L)$. In particular, we can write $U(L)=U_{0} \oplus U_{1}$, where

$$
U_{0}=\left\{u \in U(L) \mid u^{\sigma}=u\right\} \quad \text { and } \quad U_{1}=\left\{u \in U(L) \mid u^{\sigma}=-u\right\} .
$$

Furthermore, if $\alpha$ is the straightened monomial $\alpha=b_{1}^{\beta_{1}} b_{2}^{\beta_{2}} \cdots b_{n}^{\beta_{n}}$, then the parity of $\alpha$ is equal to the parity of the number of odd $b_{i}$ which actually occur in the product, that is, the number of odd $b_{i}$ with exponent equal to 1 .

Since $U(L)$ is graded, we can define [, ] on $U(L)$ as in (1) and, by the definition of $U(L)$, this agrees with the superbracket given on $L$. We therefore conclude that [, ] on $L$ extends to [, ] on $U(L)$ so that properties (1)-(5) are satisfied.

We close this section with an immediate consequence of the Poincaré-BirkhoffWitt Theorem.

Lemma 1.2. Let $L$ be a Lie superalgebra and let $H$ be a subalgebra of $L$. If $Y$ is an ordered homogeneous basis for $H$ and if $Y \cup \dot{X}$ is an ordered homogeneous basis for $L$ with $X<Y$, then every element $\alpha$ of $U(L)$ can be uniquely expressed as a finite sum $\alpha=\sum \eta \alpha_{\eta}$ where $\eta$ is a straightened monomial on the set $X$ and $\alpha_{\eta} \in U(H)$.

In the above situation, we say that $\alpha$ is written based on $H$. More generally, if $\alpha_{1}, \alpha_{2}, \ldots, \alpha_{k} \in U(L)$, then each $\alpha_{i}=\sum \eta \alpha_{i, \eta}$ is based on $H$ if the straightened monomials $\eta$ all come from the same linearly independent set $X$. 


\section{THE $\Delta$ IDEAL}

Let $L$ be a Lie superalgebra (possibly restricted) and let $H$ be a $\sigma$-stable subspace of $L$. We define

$\mathbb{D}_{L}(H)=\left\{l \in L \mid\left[H^{\prime}, l\right]=0\right.$, for some subspace $H^{\prime} \subseteq H$ of finite codimension $\}$ $=\left\{l \in L \mid \operatorname{dim}_{K}[H, l]<\infty\right\}$.

Since the intersection of a finite number of subspaces of finite codimension also has finite codimension, we immediately have

Lemma 2.1. If $V$ is a $\sigma$-stable subspace of $L$ and if $W$ is a subspace of $V$ of finite codimension, then $W \cap W^{\sigma}$ is a $\sigma$-stable subspace of $V$ of finite codimension with $W \cap W^{\sigma} \subseteq W \subseteq V$.

In particular, it follows that

$\mathbb{D}_{L}(H)=\left\{l \in L \mid\left[H^{\prime}, l\right]=0\right.$, for some $\sigma$-stable subspace $H^{\prime} \subseteq H$ of finite codimension $\}$.

Some basic properties are

Lemma 2.2. Let $H$ be a $\sigma$-stable subspace of $L$ and set $D=\mathbb{D}_{L}(H)$. Then

(i) $D$ is a Lie superalgebra of $L$.

(ii) If $L$ is restricted, then so is $D$.

(iii) If $H \triangleleft L$, then $D \triangleleft L$.

Proof. (i) It follows from (4) that $D$ is a $\sigma$-stable subspace of $L$, thus $D=$ $D_{0} \oplus D_{1}$. If $x \in D_{a}$ and $y \in D_{b}$, then since $H=H_{0} \oplus H_{1}$, the Jacobi identity yields

$$
[H,[x, y]] \subseteq[x,[H, y]]+[y,[H, x]] .
$$

Thus, since $[H, y]$ and $[H, x]$ are both finite dimensional, so is $[H,[x, y]]$, hence $[x, y] \in D$. 0 .

(ii) This follows from (7), since if $x \in D_{0}$ and $\left[x, H^{\prime}\right]=0$, then $\left[x^{[p]}, H^{\prime}\right]=$

(iii) Finally suppose $H$ is an ideal of $L$; let $x \in D_{a}$ and $l \in L_{b}$ then again

$$
[H,[x, l]] \subseteq[x,[H, l]]+[l,[H, x]]
$$

and, as above, $\operatorname{dim}_{K}[H, x]<\infty$, so $\operatorname{dim}_{K}[l,[H, x]]<\infty$. On the other hand, since $H$ is an ideal of $L$, we have $[H, l] \subseteq H$, so $\operatorname{dim}_{K}[x,[H, l]] \leq$ $\operatorname{dim}_{K}[H, x]<\infty$. Thus $\operatorname{dim}_{K}[H,[x, l]]<\infty$, so $[x, l] \in D$ and $D \triangleleft L$.

Now define the delta ideal, $\Delta(L)=\mathbb{D}_{L}(L)$, so that $\Delta(L)$ is certainly a characteristic ideal of $L$. In other words, $\Delta(L)$ is invariant under all automorphisms of $L$. It is amusing to observe

Lemma 2.3. If either $\operatorname{dim}_{K} L_{0}<\infty$ or $\operatorname{dim}_{K} L_{1}<\infty$, then $L_{1} \subseteq \Delta(L)$.

Proof. Let $x \in L_{1}$ and say $\operatorname{dim}_{K} L_{a}<\infty$; then obviously $\operatorname{dim}_{K}\left[L_{a}, x\right]<\infty$. Moreover $\left[L_{a+1}, x\right] \subseteq L_{a}$, so $\operatorname{dim}_{K}\left[L_{a+1}, x\right]<\infty$. Thus $\operatorname{dim}_{K}[L, x]<\infty$ as required.

Let $H$ be a graded subspace of $L$. Then $H$ acts on $U(L)$ and the constants for $H$ are $\{\alpha \in U(L) \mid[H, \alpha]=0\}$. The almost constants for $H$ are $\left\{\alpha \in U(L) \mid\left[H^{\prime}, \alpha\right]=0\right.$, for some subspace $H^{\prime}$ of finite codimension in $\left.H\right\}$. Note that the elements of $\mathbb{D}_{L}(H)$ are almost constants for $H$. We continue with 
Lemma 2.4. Let $H$ be a graded subspace of $L$.

(i) If $\alpha_{1}, \alpha_{2}, \ldots, \alpha_{n}$ are almost constants for $H$, then there exists a graded subspace $H^{\prime}$ of finite codimension in $H$ with $\left[H^{\prime}, \alpha_{i}\right]=0$, for all $i$.

(ii) The almost constants for $H$ are a subring of $U(L)$ which contains $U(D)$, where $D=\mathbb{D}_{L}(H)$.

Proof. Part (i) follows from Lemma 2.1 using the fact that the finite intersection of subspaces of finite codimension is of finite codimension. Let $S$ denote the almost constants for $H$. By (i) and the linearity of [ , ], it easily follows that $S$ is closed under addition. Similarly, by (i) and (5), $S$ is also closed under multiplication. Since $S \supseteq D$, we have $S \supseteq U(D)$.

If $W$ is a subspace of $V$, then a complementary basis for $V \backslash W$ is a linearly independent set $X$ such that $W \oplus K X=V$, where $K X$ is the $K$-linear span of $X$.

Now let $H$ be a graded subspace of $L$ and let $D=\mathbb{D}_{L}(H)$, so that $D$ is a (restricted) subalgebra of $L$. When writing elements of $U(L)$ based on $D$, we must, of course, choose a complementary basis for $L \backslash D$. We will, in fact, require such bases of a special type. Since $H$ is graded, we have $H=H_{0} \oplus H_{1}$, so clearly $D=\mathbb{D}_{L}\left(H_{0}\right) \cap \mathbb{D}_{L}\left(H_{1}\right)$. In addition, since both $H_{0}$ and $H_{1}$ are $\sigma$-stable, $\mathbb{D}_{L}\left(H_{0}\right)$ and $\mathbb{D}_{L}\left(H_{1}\right)$ are also both $\sigma$-stable and we have

$$
\left(\mathbb{D}_{L}\left(H_{0}\right)+\mathbb{D}_{L}\left(H_{1}\right)\right) / D=\mathbb{D}_{L}\left(H_{0}\right) / D \oplus \mathbb{D}_{L}\left(H_{1}\right) / D \text {. }
$$

It follows that we can find a homogeneous complementary basis $X$ for $L \backslash D$ such that $X=X_{0} \dot{\cup} X_{1} \dot{\cup} X_{2}$, where

$X_{0}$ is a complementary basis for $\mathbb{D}_{L}\left(H_{0}\right) \backslash D$,

$X_{1}$ is a complementary basis for $\mathbb{D}_{L}\left(H_{1}\right) \backslash D$,

$X_{2}$ is a complementary basis for $L \backslash\left(\mathbb{D}_{L}\left(H_{0}\right)+\mathbb{D}_{L}\left(H_{1}\right)\right)$.

We call such bases special and when ordering these bases, the even elements will always come first. Furthermore, notice that $X_{0} \dot{\cup} X_{2}$ is linearly independent modulo $\mathbb{D}_{L}\left(H_{1}\right)$ and $X_{1} \cup X_{2}$ is linearly independent modulo $\mathbb{D}_{L}\left(H_{0}\right)$.

If we write $\alpha \in U(L)$, as $\sum \eta \alpha_{\eta}$ based on $D$ and if we use a special basis for $L \backslash D$, then we say that $\alpha$ is specially based on $D$. This means that all monomials $\eta$ are straightened monomials in a special complementary basis for $L \backslash D$. Furthermore, we say $\alpha_{1}, \alpha_{2}, \ldots, \alpha_{n}$ are specially based on $D$ if they are all written with the same special complementary homogeneous basis for $L \backslash D$. Note that the definition of a special basis includes within it the assumption that the basis is homogeneous.

The following lemma will be crucial later in this paper.

Lemma 2.5. Let $H$ be a graded subspace of $L$ and set $D=\mathbb{D}_{L}(H)$. Suppose $Z=\left\{z_{1}, z_{2}, \ldots, z_{q}\right\}$ is a finite subset of a special complementary basis for $L \backslash D$ and let $H^{\prime}$ be a $\sigma$-stable subspace of $H$ of finite codimension. If $V$ is a finite-dimensional subspace of $L$ and if $N \geq 1$ is a positive integer, then there exist homogeneous elements $x_{i k}$, for $i=1,2, \ldots, q$ and $k=1,2, \ldots, N$ in $H^{\prime}$ such that if $t_{i j k}=\left[x_{i k}, z_{j}\right]$, then the nonzero elements in the set $\left\{t_{i j k}\right\}$ are $K$-linearly independent modulo $V$. Furthermore $t_{i i k} \neq 0$, for all $i, k$.

Proof. Since $H^{\prime}$ is of finite codimension in $H$, we have $D=\mathbb{D}_{L}\left(H^{\prime}\right)$ therefore, without loss of generality, we may assume that $H^{\prime}=H$. Furthermore, since $Z$ is a subset of a special complementary basis, we can write $Z=Z_{0} \cup Z_{1} \cup Z_{2}$ 
where $Z_{0} \subseteq \mathbb{D}_{L}\left(H_{0}\right), Z_{1} \subseteq \mathbb{D}_{L}\left(H_{1}\right), Z_{1} \cup Z_{2}$ is linearly independent modulo $\mathbb{D}_{L}\left(H_{0}\right)$ and $Z_{0} \cup Z_{2}$ is linearly independent modulo $\mathbb{D}_{L}\left(H_{1}\right)$.

Let $s=\left|Z_{1}\right|+\left|Z_{2}\right|$; we will first find $s N$ elements of $\left\{x_{i k}\right\}$ corresponding to the $z_{i} \in Z_{1} \cup Z_{2}$ and then find $(q-s) N$ elements of $\left\{x_{i k}\right\}$ corresponding to the $z_{i} \in Z_{0}$. To this end, let $H_{0}^{\prime}$ be a subspace of $H_{0}$ of finite codimension such that $\left[H_{0}^{\prime}, Z_{0}\right]=0$ and let $A$ be the $K$-linear span of $Z_{1} \dot{\cup} Z_{2}$. Since [, ] is a bilinear map from $L \times L$ to $L$, it follows that [, ]: $H_{0}^{\prime} \times A \rightarrow L$ is bilinear and no nonzero element of $A$ annihilates a subspace of $H_{0}^{\prime}$ of finite codimension. We can therefore apply Proposition 2.2 of [BP] to conclude that there exists a subspace $H_{0}^{\prime \prime}$ of $H_{0}^{\prime}$ of dimension $s N$ such that $\left[H_{0}^{\prime \prime}, A\right] \simeq H_{0}^{\prime \prime} \times A$ and $\left[H_{0}^{\prime \prime}, A\right] \cap V=0$. We then double subscript a basis for $H_{0}^{\prime \prime}$ to obtain those elements of $\left\{x_{i k}\right\}$ for which $i$ runs through all those subscripts with $z_{i} \in Z_{1} \dot{\cup} Z_{2}$ and such that $1 \leq k \leq N$. We note that all the elements of $\left\{x_{i k}\right\}$ are homogeneous and if we set $t_{i j k}=\left[x_{i k}, z_{j}\right]$ then $t_{i j k}=0$ if and only if $z_{j} \in Z_{0}$. Furthermore $\left\{t_{i j k} \mid z_{i}, z_{j} \in Z_{1} \dot{\cup} Z_{2}\right\}$ is a set of $s^{2} N$ elements which are $K$-linearly independent modulo $V$. In particular, $t_{i i k} \neq 0$ for all $z_{i} \in Z_{1} \dot{\cup} Z_{2}$.

We continue with an argument similar to the one above; let $B$ be the $K$ linear span of $Z_{0} \dot{\cup} Z_{2}$ and let $H_{1}^{\prime}$ be a subspace of $H_{1}$ of finite codimension such that $\left[H_{1}^{\prime}, Z_{1}\right]=0$. Since $[]:, H_{1}^{\prime} \times B \rightarrow L$ is bilinear and no nonzero element of $B$ annihilates a subspace of $H_{1}^{\prime}$ of finite codimension, we can again apply Proposition 2.2 of [BP]. Thus, if we let $W$ be the finite-dimensional subspace of $L$ spanned by $V$ and $\left\{t_{i j k} \mid z_{i} \in Z_{1} \cup Z_{2}\right\}$, then there exists a subspace $H_{1}^{\prime \prime}$ of $H_{1}^{\prime}$ of dimension $(q-s) N$ such that $\left[H_{1}^{\prime \prime}, B\right] \simeq H_{1}^{\prime \prime} \times B$ and $\left[H_{1}^{\prime \prime}, B\right] \cap W=0$. Now double subscript a basis for $H_{1}^{\prime \prime}$ to obtain those elements of $\left\{x_{i k}\right\}$ for which $i$ runs through all those subscripts with $z_{i} \in Z_{0}$ and such that $1 \leq k \leq N$. These new elements of $\left\{x_{i k}\right\}$ are also all homogeneous and if we set $t_{i j k}=\left[x_{i k}, z_{j}\right]$, then $t_{i j k}=0$ if $z_{j} \in Z_{1}$ and $z_{i} \in Z_{0}$. Furthermore the elements of $\left\{t_{i j k} \mid z_{i} \in Z_{0}\right.$ and $\left.z_{j} \in Z_{0} \cup Z_{2}\right\}$ are $K$-linearly independent modulo $W$ and, by the definition of $W$, it follows that the nonzero elements of $\left\{t_{i j k} \mid 1 \leq i, j \leq q\right.$ and $\left.1 \leq k \leq N\right\}$ are $K$-linearly independent modulo $V$. In particular, $t_{i i k} \neq 0$, for all $i, k$.

We now observe that $\mathbb{D}_{L}(H)$ is well behaved under field extensions.

Lemma 2.6. Let $H$ be a graded subspace of $L$ and let $F$ be an extension field of $K$. Then $F \otimes_{K} L$ is a Lie superalgebra over $F$ and

$$
\mathbb{D}_{F \otimes L}(F \otimes H)=F \otimes \mathbb{D}_{L}(H) .
$$

Proof. Set $D=\mathbb{D}_{L}(H)$; it is clear that $F \otimes D \subseteq \mathbb{D}_{F \otimes L}(F \otimes H)$. Thus for the reverse inclusion, it suffices to show that if $x_{1}, x_{2}, \ldots, x_{n} \in L$ are $K$ linearly independent modulo $D$, then they are $F$-linearly independent modulo $\mathbb{D}_{F \otimes L}(F \otimes H)$. To this end, let $B=K x_{1}+K x_{2}+\cdots+K x_{n}$ and note that $B \cap D=0$. Thus [ , ]:H $H \rightarrow L$ is bilinear and no nonzero element of $B$ annihilates a subspace of $H$ of finite codimension. Therefore, by Proposition 2.2 of [BP], there exists a finite-dimensional subspace $H^{\prime}$ of $H$, of arbitrarily large dimension over $K$, such that $\left[H^{\prime}, B\right] \simeq H^{\prime} \times B$. Therefore $\left[F \otimes H^{\prime}, F \otimes\right.$ $B] \simeq F \otimes\left(H^{\prime} \times B\right)$ and this has dimension over $F$ equal to $n\left(\operatorname{dim}_{K} H^{\prime}\right)$. However, since $\operatorname{dim}_{K} H^{\prime}$ can be chosen to be arbitrarily large, we conclude that $(F \otimes B) \cap \mathbb{D}_{F \otimes L}(F \otimes H)=0$. 
We close this section with an easy observation about $\Delta(L)$.

Lemma 2.7. Let $H$ be a finitely generated superalgebra of $\Delta(L)$. Then $H$ is constant-by-finite and $\operatorname{dim}_{K}[H, H]<\infty$.

Proof. Suppose $H$ is generated by elements $h_{1}, h_{2}, \ldots, h_{n} \in \Delta(L)$; then, by Lemma 2.4(i), there exists a graded subspace $H^{\prime}$ of finite codimension in $H$ with $\left[H^{\prime}, h_{i}\right]=0$, for all $i$. However, as in Lemma 2.4(ii), the constants for $H^{\prime}$ are a subring of $U(L)$ which contains all the $h_{i}$, hence $\left[H^{\prime}, H\right]=0$. In particular, if we let $C=\{h \in H \mid[h, H]=0\}$, then $C \supseteq H^{\prime}$ and $C$ is clearly of finite codimension in $H$. Since $C$ is the set of constants of $H$ in $H$, it follows that $H$ is constant-by-finite.

Finally, the superbracket [, ] determines an epimorphism [, ]:H/C $\times$ $H / C \rightarrow[H, H]$. Since $\operatorname{dim}_{K} H / C<\infty$, we conclude that $\operatorname{dim}_{K}[H, H]<\infty$.

\section{DeRivation IDENTITIES}

Let $x \in L_{c}$, let $\partial(x): U(L) \rightarrow U(L)$ defined by $\partial(x)(\alpha)=[x, \alpha]$, for all $\alpha \in U(L)$, be the superderivation induced by $x$. We will write the image of $\alpha$ as $\alpha^{x}$. Some basic properties of $\partial(x)$ are as follows:

(1') $\alpha^{x}=x \alpha-(-1)^{a c} \alpha x \in U_{a+c}$, for all $\alpha \in U_{a}$, and, by (5), it follows that

(5') $(\alpha \beta)^{x}=\alpha^{x} \beta+(-1)^{a c} \alpha \beta^{x}$, for all $\alpha \in U_{a}$ and $\beta \in U_{b}$.

Throughout, if $\alpha$ is a homogeneous element of $U(L)$, we will let $\varepsilon(\alpha)$ denote the parity of $\alpha$.

Lemma 3.1. Let $\alpha_{1}, \alpha_{2}, \ldots, \alpha_{n}$ be homogeneous elements of $U(L)$ and let $x$ be a homogeneous element of $L$. Then

$$
\left(\alpha_{1} \alpha_{2} \cdots \alpha_{n}\right)^{x}=\sum_{i}(-1)^{\varepsilon(x) \varepsilon\left(\alpha_{1} \alpha_{2} \cdots \alpha_{i-1}\right)} \alpha_{1} \alpha_{2} \cdots \alpha_{i-1} \alpha_{i}^{x} \alpha_{i+1} \cdots \alpha_{n}
$$

In particular, if $\alpha \in U(L)$, then $\operatorname{deg} \alpha^{x} \leq \operatorname{deg} \alpha$.

Proof. The first formula follows by applying induction to $\left(5^{\prime}\right)$. The degree formula follows by writing $\alpha$ in terms of straightened monomials and applying the superderivation induced by $x$ to each of the monomials. By the first formula, no monomial appearing in $\alpha^{x}$ will have degree exceeding $\operatorname{deg} \alpha$.

Our next lemma easily follows from Lemma 3.1 .

Lemma 3.2. Let $\alpha_{1}, \alpha_{2}, \ldots, \alpha_{n}$ be homogeneous elements of $U(L)$ and let $x_{1}, x_{2}, \ldots, x_{n}$ be homogeneous elements of $L$. Then

$$
\begin{aligned}
\left(\alpha_{1} \alpha_{2} \cdots \alpha_{n}\right)^{x_{n} x_{n-1} \cdots x_{1}}= & \sum_{\sigma \in \operatorname{Sym}_{n}} \pm \alpha_{1}^{x_{\sigma_{1}}} \alpha_{2}^{x_{\sigma_{2}}} \cdots \alpha_{n}^{x_{\sigma_{n}}} \\
& + \text { terms with at least one } \alpha_{i} \text { not differentiated } .
\end{aligned}
$$

It is a thankless task to compute the individual + and - signs in the above lemma, but fortunately computing them will not be necessary.

Lemma 3.3. Let $\alpha_{1}, \alpha_{2}, \ldots, \alpha_{n}$ be even elements of $U(L)$, let $\beta_{1}, \beta_{2}, \ldots, \beta_{m}$ be homogeneous elements of $U(L)$, and let $x_{1}, x_{2}, \ldots, x_{n}, y_{1}, y_{2}, \ldots, y_{m}$ be homogeneous elements of $L$. Set $k=\operatorname{deg} \alpha_{1}+\operatorname{deg} \alpha_{2}+\cdots+\operatorname{deg} \alpha_{n}+\operatorname{deg} \beta_{1}+$ 
$\operatorname{deg} \beta_{2}+\cdots+\operatorname{deg} \beta_{m}$. Then

$$
\begin{aligned}
\left(\alpha_{1} \alpha_{2} \cdots \alpha_{n} \beta_{1} \beta_{2} \cdots \beta_{m}\right)^{x_{n} x_{n-1} \cdots x_{1} y_{m} y_{m-1} \cdots y_{1}} \\
=\left(\sum_{\sigma \in \mathrm{Sym}_{n}} \alpha_{\sigma_{1}}^{x_{1}} \alpha_{\sigma_{2}}^{x_{2}} \cdots \alpha_{\sigma_{n}}^{x_{n}}\right)\left(\sum_{\tau \in \mathrm{Sym}_{m}} \pm \beta_{\tau_{1}}^{y_{1}} \beta_{\tau_{2}}^{y_{2}} \cdots \beta_{\tau_{m}}^{y_{m}}\right) \\
\quad+\text { terms with at least one } \alpha_{i} \text { or } \beta_{j} \text { not differentiated } \\
\quad+\text { terms of degree less than } k \\
\quad+\text { terms with at least one } \alpha_{i} \text { differentiated by some } y_{j} .
\end{aligned}
$$

Proof. Let $M$ denote the $K$-linear span of all possible terms in the last three categories listed above. The goal is to compute

$$
\left(\alpha_{1} \alpha_{2} \cdots \alpha_{n} \beta_{1} \beta_{2} \cdots \beta_{m}\right)^{x_{n} x_{n-1} \cdots x_{1} y_{m} y_{m-1} \cdots y_{1}}
$$

modulo $M$. To this end, we differentiate $\alpha_{1} \alpha_{2} \cdots \alpha_{n} \beta_{1} \beta_{2} \cdots \beta_{m}$ by each $x_{i}$ and $y_{j}$ in turn. In the course of this procedure certain monomials occur which may not yet be in $M$, but which will necessarily be in $M$ once all the $n+m$ differentiations are applied. Thus we use $\equiv$ to denote not merely congruence modulo $M$, but rather eventual congruence modulo $M$. For example, any monomial containing a factor $\beta_{j}^{x_{i}}$ or a second derivative is eventually in $M$ since, when all differentiations are applied, it is clear that either some $a_{l}$ is not differentiated or it is differentiated by some $y_{s}$. In particular, we can view each $\beta_{j}$ and each first derivative as a constant for $x_{i}$ modulo $M$.

We begin by applying $x_{n}$. Since modulo $M$ all the $\beta_{j}$ are constants and since all the $\alpha_{i}$ are even, we get

$$
\left(\alpha_{1} \alpha_{2} \cdots \alpha_{n} \beta_{1} \beta_{2} \cdots \beta_{m}\right)^{x_{n}} \equiv\left(\sum_{i=1}^{n} \alpha_{1} \cdots \alpha_{i}^{x_{n}} \cdots \alpha_{n}\right) \beta_{1} \beta_{2} \cdots \beta_{m} .
$$

Although $\alpha_{i}^{x_{n}}$ may not be even, all the $\alpha_{j}$ are even and therefore we can move $\alpha_{i}^{x_{n}}$ to the right of $\alpha_{n}$ modulo terms of degree less than $k$. Thus

$$
\left(\alpha_{1} \alpha_{2} \cdots \alpha_{n} \beta_{1} \beta_{2} \cdots \beta_{m}\right)^{x_{n}} \equiv \sum_{t_{n}} \alpha_{1} \cdots \hat{\alpha}_{t_{n}} \cdots \alpha_{n} \alpha_{t_{n}}^{x_{n}} \beta_{1} \beta_{2} \cdots \beta_{m},
$$

where " indicates that the factor is missing.

Next we apply $x_{n-1}$; once again we view $\beta_{1}, \beta_{2}, \ldots, \beta_{m}$ as constants modulo $M$. In addition, we can also view $\alpha_{t_{n}}^{x_{n}}$ as a constant modulo $M$, since if $\alpha_{t_{n}}^{x_{n}}$ is differentiated more than once, then some $\alpha$ or $\beta$ factor will not be differentiated. Since every $\alpha$ is even, this gives us

$$
\begin{aligned}
& \left(\alpha_{1} \alpha_{2} \cdots \alpha_{n} \beta_{1} \beta_{2} \cdots \beta_{m}\right)^{x_{n} x_{n-1}} \\
& \equiv \sum_{t_{n}}\left(\sum_{\substack{i=1 \\
i \neq l_{n}}} \alpha_{1} \cdots \alpha_{i}^{x_{n-1}} \cdots \hat{\alpha}_{t_{n}} \cdots \alpha_{n}\right) \alpha_{t_{n}}^{x_{n}} \beta_{1} \beta_{2} \cdots \beta_{m} .
\end{aligned}
$$

Again, since each $\alpha$ is even, we can slide $\alpha_{i}^{x_{n-1}}$ to the right of $\alpha_{n}$, modulo terms of degree less than $k$. Thus

$$
\begin{aligned}
& \left(\alpha_{1} \alpha_{2} \cdots \alpha_{n} \beta_{1} \beta_{2} \cdots \beta_{m}\right)^{x_{n} x_{n-1}} \\
& \quad \equiv \sum_{t_{n-1} \neq t_{n}} \alpha_{1} \cdots \hat{\alpha}_{t_{n-1}} \cdots \hat{\alpha}_{t_{n}} \cdots \alpha_{n} \alpha_{t_{n-1}}^{x_{n-1}} \alpha_{t_{n}}^{x_{n}} \beta_{1} \beta_{2} \cdots \beta_{m} .
\end{aligned}
$$


Since $t_{n-1} \neq t_{n}$, the above sum is taken over the start of a permutation in $\mathrm{Sym}_{n}$. Continuing in this manner with the other $x_{i}$, we clearly obtain

$$
\begin{aligned}
& \left(\alpha_{1} \alpha_{2} \cdots \alpha_{n} \beta_{1} \beta_{2} \cdots \beta_{m}\right)^{x_{n} x_{n-1} \cdots x_{1}} \\
& \quad \equiv\left(\sum_{\sigma \in \operatorname{Sym}_{n}} \alpha_{\sigma_{1}}^{x_{1}} \alpha_{\sigma_{2}}^{x_{2}} \cdots \alpha_{\sigma_{n}}^{x_{n}}\right) \beta_{1} \beta_{2} \cdots \beta_{m} .
\end{aligned}
$$

Now set $A=\sum_{\sigma \in \operatorname{Sym}_{n}} \alpha_{\sigma_{1}}^{x_{1}} \alpha_{\sigma_{2}}^{x_{2}} \cdots \alpha_{\sigma_{n}}^{x_{n}}$ and notice that $A$ is homogeneous since each summand has parity $\varepsilon\left(x_{1}\right)+\varepsilon\left(x_{2}\right)+\cdots+\varepsilon\left(x_{n}\right)$. Moreover, $\beta_{1} \beta_{2} \cdots \beta_{m}$ is also homogeneous and thus we have

$$
A\left(\beta_{1} \beta_{2} \cdots \beta_{m}\right)= \pm\left(\beta_{1} \beta_{2} \cdots \beta_{m}\right) A+\text { terms of degree less than } k .
$$

Finally, we must compute $\left( \pm \beta_{1} \beta_{2} \cdots \beta_{m} A\right)^{y_{m} y_{m-1} \cdots y_{1}}$ modulo $M$. In this case, we can assume that $A$ is a constant for each $y_{j}$, since otherwise second or higher order derivatives occur. Thus, by Lemma 3.2, we have

$$
\left( \pm \beta_{1} \beta_{2} \cdots \beta_{m} A\right)^{y_{m} y_{m-1} \cdots y_{1}} \equiv\left(\sum_{\tau \in \mathrm{Sym}_{m}} \pm \beta_{1}^{y_{\tau_{1}}} \beta_{2}^{y_{\tau_{2}}} \cdots \beta_{m}^{y_{\tau}}\right) A .
$$

Since each $\beta_{j}^{y_{\tau_{j}}}$ is homogeneous, we can interchange these factors up to a + or - sign and terms of lower degree. Specifically, we do these interchanges so that they $y_{i}$ 's are written in the correct order. This yields

$$
\sum_{\tau \in \mathrm{Sym}_{m}} \pm \beta_{1}^{y_{\tau_{1}}} \beta_{2}^{y_{\tau_{2}}} \cdots \beta_{m}^{y_{\tau_{m}}}=\left(\sum_{\tau \in \mathrm{Sym}_{m}} \pm \beta_{\tau_{1}}^{y_{1}} \beta_{\tau_{2}}^{y_{2}} \cdots \beta_{\tau_{m}}^{y_{m}}\right)+\text { lower degree terms, }
$$

where, of course, the individual \pm signs on the right may certainly differ from those on the left. Thus if we set $B=\left(\sum_{\tau \in \mathrm{Sym}_{m}} \pm \beta_{\tau_{1}}^{y_{1}} \beta_{\tau_{2}}^{y_{2}} \cdots \beta_{\tau_{m}}^{y_{m}}\right)$, then

$$
\left( \pm \beta_{1} \beta_{2} \cdots \beta_{m} A\right)^{y_{m} y_{m-1} \cdots y_{1}} \equiv\left(\sum_{\tau \in \mathrm{Sym}_{m}} \pm \beta_{\tau_{1}}^{y_{1}} \beta_{\tau_{2}}^{y_{2}} \cdots \beta_{\tau_{m}}^{y_{m}}\right) A=B A .
$$

Now note that $B$ is homogeneous since each summand has parity $\varepsilon\left(\beta_{1}\right)+$ $\cdots+\varepsilon\left(\beta_{m}\right)+\varepsilon\left(y_{1}\right)+\cdots+\varepsilon\left(y_{m}\right)$. Thus, modulo terms of degree less than $k$, we have $B A= \pm A B$. By absorbing the + or - into the $B$ summand, we obtain

$$
\left(\alpha_{1} \alpha_{2} \cdots \alpha_{n} \beta_{1} \beta_{2} \cdots \beta_{m}\right)^{x_{n} x_{n-1} \cdots x_{1} y_{m} y_{m-1} \cdots y_{1}} \equiv A( \pm B),
$$

thereby proving the lemma.

If $X$ is a subset of $L$, recall that $X^{n}$ is the $K$-linear span of all products of the form $x_{1} x_{2} \cdots x_{k}$ with $x_{i} \in X$ and $0 \leq k \leq n$. We continue with

Lemma 3.4. Let $Z=\left\{z_{1}, z_{2}, \ldots, z_{q}\right\}$ be a homogeneous linearly independent subset of $L$ written with all the even terms coming first and let $\xi=z_{1}^{b_{1}} z_{2}^{b_{2}} \cdots z_{q}^{b_{q}}$ be a straightened monomial in $U(L)$ of degree $n=b_{1}+b_{2}+\cdots+b_{q}$.

Let $\left\{x_{i j} \mid 1 \leq i \leq q\right.$ and $\left.1 \leq j \leq n^{\prime}\right\}$ be a set of homogeneous elements in $L$, with $n^{\prime} \geq n$ and set $t_{i j k}=\left[x_{i k}, z_{j}\right]$. Assume that the nonzero elements in $T=\left\{t_{i j k}\right\}$ are linearly independent, order them lexicographically, and also assume that $t_{i i k} \neq 0$, for all $i, k$. 
Now let $\delta=x_{1,1} \cdots x_{1, a_{1}} x_{2,1} \cdots x_{2, a_{2}} \cdots x_{q, 1} \cdots x_{q, a_{q}}$, with $0 \leq a_{i}$ and $a_{1}+$ $a_{2}+\cdots+a_{q}=n$. Then $\xi^{\delta}=\xi_{1}+\xi_{2}+\xi_{3}$, where $\xi_{1} \in \sum_{s=0}^{n-1} L^{s} Z^{1} L^{n-s-1}, \xi_{2}$ is a $K$-linear combination of straightened monomials of degree $n$ in the $t_{i j k}$ having at least one factor $t_{i j k}$ with $i \neq j$, and finally,

$$
\xi_{3}= \pm a_{1} ! a_{2} ! \cdots a_{q} ! t_{1,1,1} \cdots t_{1,1, q_{1}} t_{2,2,1} \cdots t_{2,2, a_{2}} \cdots t_{q, q, 1} \cdots t_{q, q, a_{q}},
$$

if $a_{i}=b_{i}$ for all $i$, but $\xi_{3}=0$ otherwise.

Proof. Since $n^{\prime} \geq n \geq a_{i}$, for every $i$, there are sufficiently many $x_{i j}$ available to form $\delta$. In addition, since $T$ is a set of ordered, homogeneous, $K$-linearly independent elements of $L$, we can consider $T$ to be part of an ordered basis of $L$.

Each $z_{j}$ is homogeneous, therefore we can begin by viewing $\xi$ as a product of $n$ homogeneous elements and then use Lemma 3.2. If some monomial in $\xi^{\delta}$ contains a $z_{j}$ factor which is not differentiated, then that monomial is contained in $\sum_{s=0}^{n-1} L^{s} Z^{1} L^{n-s-1}$ and absorbs into $\xi_{1}$. On the other hand, if every $z_{j}$ factor of a monomial is differentiated by some $x_{i k}$, we obtain \pm a product of various $t_{i j k}$. Notice that no $t_{i j k}$ occurs more than once, since each $x_{i k}$ occurs at most once in $\delta$. Monomials, all of whose factors belong to $T$, can then be straightened modulo terms of degree less than $n$ which absorb into $\xi_{1}$. Thus $\xi^{\delta}$ is indeed of the form $\xi_{1}+\xi_{2}+\xi_{3}$, where $\xi_{3}$ is the sum of all straightened monomials in the various $t_{i i k}$. It remains to compute $\xi_{3}$.

The $\xi_{3}$ term occurs only if each $z_{j}$ is differentiated by some $x_{i k}$ with $j=i$. Clearly, this can only happen if $a_{i}=b_{i}$, for all $i$, therefore we will now assume that this is the case. Say $z_{1}, z_{2}, \ldots, z_{e}$ are the even elements of $Z$ and $z_{e+1}, \ldots, z_{q}$ are all odd. Write

$$
\xi=z_{1}^{a_{1}} z_{2}^{a_{2}} \cdots z_{q}^{a_{q}}=\alpha_{1} \alpha_{2} \cdots \alpha_{r} \beta_{1} \beta_{2} \cdots \beta_{s},
$$

where each $\alpha_{i}$ is some $z_{i^{\prime}}$ with $i^{\prime} \leq e$ and each $\beta_{j}$ is some $z_{i^{\prime \prime}}$ with $i^{\prime \prime}>e$. Furthermore, write

$$
\delta=u_{1} u_{2} \cdots u_{r} v_{1} v_{2} \cdots v_{s}
$$

where each $u_{i}$ is some $x_{i^{\prime}, j^{\prime}}$ with $i^{\prime} \leq e$ and each $v_{i}$ is some $x_{i^{\prime \prime}, j^{\prime \prime}}$ with $i^{\prime \prime}>e$. We now apply Lemma 3.3 to

$$
\xi^{\delta}=\left(\alpha_{1} \alpha_{2} \cdots \alpha_{r} \beta_{1} \beta_{2} \cdots \beta_{s}\right)^{u_{1} u_{2} \cdots u_{r} v_{1} v_{2} \cdots v_{s}} .
$$

Notice that the unspecified summands in Lemma 3.3 all absorb into $\xi_{1}$ or $\xi_{2}$. Thus the $\xi_{3}$ term will come from

$$
A B=\left(\sum_{\sigma \in \mathrm{Sym}_{r}} \alpha_{\sigma_{1}}^{u_{r}} \alpha_{\sigma_{2}}^{u_{r-1}} \cdots \alpha_{\sigma_{r}}^{u_{1}}\right)\left(\sum_{\tau \in \mathrm{Sym}_{s}} \pm \beta_{\tau_{1}}^{v_{s}} \beta_{\tau_{2}}^{v_{s-1}} \cdots \beta_{\tau_{s}}^{v_{1}}\right) .
$$

We get a $\xi_{3}$ contribution if and only if $\sigma$ and $\tau$ are chosen so that the $z$ subscript of $\alpha_{\sigma_{i}}$ matches the first $x$ subscript of $u_{r-i+1}$ and similarily, the $z$ subscript of $\beta_{\tau_{j}}$ matches the first $x$ subscript of $v_{s-j+1}$. Since $a_{i}=b_{i}$, all such summands in the $A$ factor must therefore look like

$$
z_{e}^{x_{e, a_{e}}} \cdots z_{e}^{x_{e, 1}} \cdots z_{2}^{x_{2, a_{2}}} \cdots z_{2}^{x_{2,1}} z_{1}^{x_{1, a_{1}}} \cdots z_{1}^{x_{1,1}},
$$

and there are precisely $a_{1} ! a_{2} ! \cdots a_{e}$ ! permutations of the $\alpha_{i}$ 's which leave the $z_{i}$ 's in this order. Thus the contribution to $\xi_{3}$ from $A$ is

$$
a_{1} ! a_{2} ! \cdots a_{e} ! t_{e, e, a_{e}} \cdots t_{e, e, 1} \cdots t_{2,2, a_{2}} \cdots t_{2,2,1} t_{1,1, a_{1}} \cdots t_{1,1,1} \text {. }
$$


For $B$, since the corresponding $z_{i}$ 's are all odd, we must have for each $i$, $a_{i}=b_{i}=0$ or 1 . Therefore $a_{i} !=1$ and there is only one possible permutation of the $\beta$ 's which yields a contribution. Indeed this contribution is

$$
z_{q}^{x_{q, a_{q}}} z_{q-1}^{x_{q-1, a_{q-1}}} \cdots z_{e+1}^{x_{e+1, a_{e+1}}}= \pm t_{q, q, a_{q}} t_{q-1, q-1, a_{q-1}} \cdots t_{e+1, e+1, a_{e+1}},
$$

with the understanding that if $a_{i}=0$ then the term is not present. Thus the contribution to $\xi_{3}$ from $A B$ is

$$
\pm a_{1} ! a_{2} ! \cdots a_{q} ! t_{e, e, a_{e}} \cdots t_{e, e, 1} \cdots t_{1,1, a_{1}} \cdots t_{1,1,1} t_{q, q, a_{q}} \cdots t_{e+1, e+1, a_{e+1}} .
$$

Finally, all the $t_{i j k}$ are homogeneous, thus they either commute or anticommute modulo lower degree terms which absorb into $\xi_{1}$. Therefore we get

$$
\xi_{3}= \pm a_{1} ! a_{2} ! \cdots a_{q} ! t_{1,1,1} \cdots t_{1,1, a_{1}} t_{2,2,1} \cdots t_{2,2, a_{2}} \cdots t_{q, q, 1} \cdots t_{q, q, a_{q}}
$$

as required and the lemma is proved.

Let us recall that if $x \in L_{a}$, then $\partial(x): U(L) \rightarrow U(L)$ is the superderivation given by $\partial(x)(\alpha)=[x, \alpha]$, for all $\alpha \in U(L)$. If $X$ is any $\sigma$-stable subspace of $L$, let $\partial^{n}(X)$ be the set of all $n$-fold derivations $\partial\left(x_{1}\right) \partial\left(x_{2}\right) \cdots \partial\left(x_{n}\right)$ with each $x_{i}$ homogeneous in $X$. Here $\partial^{0}(X)=\{1\}$ and we let $\partial^{\infty}(X)=\bigcup_{n=0}^{\infty} \partial^{n}(X)$.

Proposition 3.5. Let $H$ be a $\sigma$-stable subalgebra of $L$ and set $D=\mathbb{D}_{L}(H)$. Fix a special complementary basis for $L \backslash D$ and assume that all $\eta$ 's below are straightened monomials in that basis. For each of these finitely many distinct $\eta$, let $\gamma_{\eta} \in U(L)$ and assume that integers $r, s$ are given such that

(i) all $\eta$ have degree $\leq r$,

(ii) $\operatorname{deg} \sum_{\eta} \eta^{\delta} \gamma_{\eta} \leq s$, for all $\delta \in \partial^{r}\left(H^{\prime}\right)$,

where $H^{\prime}$ is a graded subspace of $H$ of finite codimension. Then for all $\eta$ we have $\operatorname{deg} \eta+\operatorname{deg} \gamma_{\eta} \leq s$. Here, of course, $\operatorname{deg} 0=-\infty$.

Proof. Let $N$ be the maximum of $\operatorname{deg} \eta+\operatorname{deg} \gamma_{\eta}$, taken over all $\eta$, and assume, by way of contradiction, that $N>s$. Among all such terms with $\operatorname{deg} \eta+\operatorname{deg} \gamma_{\eta}=$ $N$, suppose the monomial $\eta=\xi$ has maximal degree $n$. Thus $n \leq r$.

By assumption, each $\eta$ is a monomial in the set $Z=\left\{z_{1}, z_{2}, \ldots, z_{q}\right\}$ which is a finite subset of a special complementary basis for $L \backslash D$. In particular, $Z$ consists of homogeneous elements and is ordered so that the even members come first. Now choose $Y \supseteq Z$ to be a finite, ordered, linearly independent, homogeneous subset of $L$ such that all $\gamma_{\eta}$ can be written in terms of the basis elements in $Y$.

Since $Z=\left\{z_{1}, z_{2}, \ldots, z_{q}\right\}$ is $K$-linearly independent modulo $D=\mathbb{D}_{L}(H)$ and since $H^{\prime}$ is of finite codimension in $H$, it follows from Lemma 2.5 that there exist homogeneous $\left\{x_{i k} \mid 1 \leq i \leq q\right.$ and $\left.1 \leq k \leq N\right\} \subseteq H^{\prime}$ such that if $t_{i j k}=\left[x_{i k}, z_{j}\right]$, then the nonzero elements of the set $\left\{t_{i j k}\right\}$ are $K$-linearly independent modulo the finite-dimensional subspace spanned by $Y$. Furthermore, $t_{i i k} \neq 0$, for all $i, k$.

The $t_{i j k}$ of finite are all homogeneous, since the $z_{j}$ and the $x_{i k}$ are homogeneous. Now order the nonzero $t_{i j k}$ lexicographically and then extend $\left\{t_{i j k}\right\}$ to a set $T$ so that $B=T \cup Y$ is a homogeneous, ordered basis for $L$ with $T<Y$.

Write $\xi=z_{1}^{a_{1}} z_{2}^{a_{2}} \cdots z_{q}^{a_{q}}$, with $a_{1}+a_{2}+\cdots+a_{q}=\operatorname{deg} \xi=n$, and let $\delta$ be the $n$-fold derivation given by

$$
\begin{aligned}
\delta & =\partial\left(x_{1,1}\right) \cdots \partial\left(x_{1, a_{1}}\right) \partial\left(x_{2,1}\right) \cdots \partial\left(x_{2, a_{2}}\right) \cdots \partial\left(x_{q, 1}\right) \cdots \partial\left(x_{q, a_{q}}\right) \\
& \in \partial^{n}\left(H^{\prime}\right) \subseteq \partial^{r}\left(H^{\prime}\right) .
\end{aligned}
$$


We now examine the degree $N$ terms of $\sum \eta^{\delta} \gamma_{\eta}$. Let

$$
\tau=t_{1,1,1} \cdots t_{1,1, a_{1}} t_{2,2,1} \cdots t_{2,2, a_{2}} \cdots t_{q, q, 1} \cdots t_{q, q, a_{q}},
$$

and we specifically consider the degree $N$ terms of $\sum \eta^{\delta} \gamma_{\eta}$ contained in $\tau Y^{N-n}$. Note that $\tau \neq 0$, since $t_{i i k} \neq 0$, for all $i, k$.

If $\operatorname{deg} \eta+\operatorname{deg} \gamma_{\eta}<N$, then the term $\eta^{\delta} \gamma_{\eta}$ has degree less than $N$ and therefore makes no contribution. Thus suppose $\operatorname{deg} \eta+\operatorname{deg} \gamma_{\eta}=N$; if $\operatorname{deg} \eta<n$ then $\eta^{\delta} \gamma_{\eta} \subseteq L^{n-1} Y^{N-n+1}$ and the latter set is disjoint from $\tau Y^{N-n}$. This follows since the degree $N$ monomials in $L^{n-1} Y^{N-n+1}=\left(T^{1}+Y^{1}\right)^{n-1} Y^{N-n+1}$ have less than $n$ factors from $T$. Therefore we may also suppose that $\operatorname{deg} \eta=$ $n$; thus $\eta=z_{1}^{b_{1}} z_{2}^{b_{2}} \cdots z_{q}^{b_{q}}$ with $b_{1}+b_{2}+\cdots+b_{q}=n$. By Lemma $3.4, \eta^{\delta}=$ $\eta_{1}+\eta_{2}+\eta_{3}$, where $\eta_{1} \in \sum_{s=0}^{n-1} L^{s} Z^{1} L^{n-s-1} \subseteq \sum_{s=0}^{n-1} L^{s} Y^{1} L^{n-s-1}$ and $\eta_{2}$ is a $K$-linear combination of straightened monomials of degree $n$ in the $t_{i j k}$ having at least one factor $t_{i j k}$ with $i \neq j$.

Note that $\eta_{1} \gamma_{\eta} \in \sum_{s=0}^{n-1} L^{s} Y^{1} L^{n-s-1} Y^{N-n} \subseteq L^{n-1} Y^{N-n+1}$ and hence $\eta_{1} \gamma_{\eta}$ makes no contribution to the terms in $\tau Y^{N-n}$. Similarly, from the form of $\eta_{2}$, we see that $\eta_{2} \gamma_{\eta} \subseteq \eta_{2} Y^{N-n}$ also makes no degree $N$ contribution to the terms in $\tau Y^{N-n}$. Therefore the only possible contribution to $\tau Y^{N-n}$ must come from $\eta_{3}$. However, by Lemma 3.4, $\eta_{3}=0$ unless $\eta=\xi$.

It now follows that the unique contribution to $\tau Y^{N-n}$ of degree $N$ is $\xi_{3} \gamma_{\xi}^{\prime}$, where $\gamma_{\xi}^{\prime}$ is the degree $N-n$ part of $\gamma_{\xi}$. However, $\gamma_{\xi}^{\prime}$ is a nonzero element of $Y^{N-n}$ and $\xi_{3}= \pm a_{1} ! a_{2} ! \cdots a_{q} ! \tau$. Moreover, if char $K=p>2$ then $a_{i}<p$, for all $i$ including the odd terms, so $\xi_{3} \neq 0$. Thus we obtain a unique degree $N$ contribution to $\tau Y^{N-n}$, contradicting the assumption that $\operatorname{deg} \sum \eta^{\delta} \gamma_{\eta} \leq s<$ $N$. We therefore conclude that $\operatorname{deg} \eta+\operatorname{deg} \gamma_{\eta} \leq s$, for all $\eta$.

The identity in the statement of the next result is called a derivation identity.

Theorem 3.6. Let $H$ be a graded subspace of $L$ and suppose either char $K=0$ or char $K=p>2$ and $L$ is restricted. Let $\alpha_{i}, \beta_{i} \in U(L)$ and assume that

$$
\alpha_{1}^{\delta} \beta_{1}+\alpha_{2}^{\delta} \beta_{2}+\cdots+\alpha_{v}^{\delta} \beta_{v}=0 \quad \text { for all } \delta \in \partial^{\infty}\left(H^{\prime}\right)
$$

where $H^{\prime}$ is a graded subspace of $H$ of finite codimension.

Write each $\alpha_{i}=\sum_{\eta} \eta \alpha_{\eta, i}$ specially based on $D=\mathbb{D}_{L}(H)$. Then, for all $\eta$, we have

$$
\alpha_{\eta, 1} \beta_{1}+\alpha_{\eta, 2} \beta_{2}+\cdots+\alpha_{\eta, v} \beta_{v}=0 .
$$

In particular, if some $\alpha_{i}$ is not zero, then $\left\{\beta_{1}, \beta_{2}, \ldots, \beta_{v}\right\}$ is left linearly dependent over $U(D)$.

Proof. Define $\gamma_{\eta}=\alpha_{\eta, 1} \beta_{1}+\alpha_{\eta, 2} \beta_{2}+\cdots+\alpha_{\eta, v} \beta_{v}$, for each $\eta$. Since $\alpha_{\eta, i} \in$ $U(D)$, it follows from Lemma 2.4 that there exists a graded subspace $H^{\prime \prime}$ of finite codimension in $H$ with $\left[H^{\prime \prime}, \alpha_{\eta, i}\right]=0$, for all $\eta, i$. Replacing $H^{\prime}$ by $H^{\prime} \cap H^{\prime \prime}$, we can assume that $\left[H^{\prime}, \alpha_{\eta, i}\right]=0$, for all $\eta, i$. It follows from Lemma 1.1 (ii) and the fact that $H^{\prime}$ is graded, that $\left(\eta \alpha_{\eta, i}\right)^{\delta}=\eta^{\delta} \alpha_{\eta, i}$, for all $\delta \in \partial^{\infty}\left(H^{\prime}\right)$. Hence the derivation identity becomes

$$
0=\sum_{\eta, i}\left(\eta \alpha_{\eta, i}\right)^{\delta} \beta_{i}=\sum_{\eta, i} \eta^{\delta} \alpha_{\eta, i} \beta_{i}=\sum_{\eta} \eta^{\delta} \gamma_{\eta} .
$$

The result now follows from Proposition 3.5 with $r=\max (\operatorname{deg} \eta)$ and $s=-1$. 


\section{LINEAR IDENTITIES}

Let $R$ be any ring; a linear identity in $R$ is any equation of the form

$$
\alpha_{1} r \beta_{1}+\alpha_{2} r \beta_{2}+\cdots+\alpha_{v} r \beta_{v}=0,
$$

which holds for all $r \in \widetilde{R}$. Here each $\alpha_{i}$ and $\beta_{i}$ is a fixed element of $R$ and $\widetilde{R}$ is some "large" subset of the ring. From a ring theoretic point of view, linear identities are more important than derivation identities, as they arise in numerous ring theoretic situations. In dealing with such identities in $R=U(L)$, there is no harm in assuming that the $\alpha$ 's and $\beta$ 's homogeneous. Our results here will not be quite as sharp as those obtained in the previous section for derivation identities.

Our main assumptions remain in force. Thus either char $K=0$ or char $K=$ $p>2$ with $L$ restricted and, depending upon char $K, U(L)$ has as a $K$ basis the monomials described in either the Poincaré-Birkhoff-Witt Theorem or Jacobson's Theorem. We start with the easier homogeneous case (assumptions (i) or (ii) below). Recall that if $X$ is a subset of $L$, then $X^{\infty}=\bigcup_{n=0}^{\infty} X^{n}$.

Theorem 4.1. Let $H$ be a graded subspace of $L$. Let $\alpha_{i}, \beta_{i} \in U(L)$ and assume that

$$
\alpha_{1} x \beta_{1}+\alpha_{2} x \beta_{2}+\cdots+\alpha_{v} x \beta_{v}=0 \quad \text { for all } x \in\left(H^{\prime}\right)^{\infty},
$$

whee $H^{\prime}$ is a graded subspace of $H$ of finite codimension. Write each $\alpha_{i}=$ $\sum_{\eta} \eta \alpha_{\eta, i}$ specially based on $D=\mathbb{D}_{L}(H)$.

Now assume that either

(i) all $\alpha_{i}$ are homogeneous of the same parity, or

(ii) $\operatorname{dim}_{K} H_{1}<\infty$.

Then, for all $\eta$, we have

$$
\alpha_{\eta, 1} \beta_{1}+\alpha_{\eta, 2} \beta_{2}+\cdots+\alpha_{\eta, v} \beta_{v}=0 .
$$

In particular, if some $\alpha_{i}$ is not zero, then $\left\{\beta_{1}, \beta_{2}, \ldots, \beta_{v}\right\}$ is left linearly dependent over $U(D)$.

Proof. Since $\mathbb{D}_{L}(H)=\mathbb{D}_{L}\left(H^{\prime}\right)$, we can assume that $H^{\prime}=H$. Also if $H_{1}$ is finite dimensional, then $\operatorname{dim}_{K}\left(H / H_{0}\right)<\infty$ and we can further assume that $H=H_{0}$. Let $x \in H^{\infty}$ and let $h$ be a homogeneous element of $H$; since $h x \in H^{\infty}$ we have

$$
\sum_{i} \alpha_{i} h x \beta_{i}=0=\sum_{i} h \alpha_{i} x \beta_{i} \quad \text { for all } x \in H^{\infty} .
$$

Now, by (i), all the $\alpha_{i}$ are homogeneous of the same parity or, by (ii), all the $h$ are even. Therefore there exists a sign $(-1)^{a}$ such that $\alpha_{i}^{h}=h \alpha_{i}-(-1)^{a} \alpha_{i} h$, for all $i$. Hence the above displayed equations yield

$$
0=\sum_{i}\left(h \alpha_{i}-(-1)^{a} \alpha_{i} h\right) x \beta_{i}=\sum_{i} \alpha_{i}^{h} x \beta_{i} .
$$

Notice also in case (i) that we have $\varepsilon\left(\alpha_{i}^{h}\right)=\varepsilon\left(\alpha_{i}\right)+\varepsilon(h)$, so that if all the $\alpha_{i}$ have the same parity, then the same is true for the $\alpha_{i}^{h}$. We can therefore continue in this manner to conclude that

$$
0=\sum_{i} \alpha_{i}^{\delta} x \beta_{i} \quad \text { for all } x \in H^{\infty} \text { and } \delta \in \partial^{\infty}(H) \text {. }
$$


In particular, with $x=1$, we obtain the derivation identity

$$
0=\sum_{i} \alpha_{i}^{\delta} \beta_{i} \quad \text { for all } \delta \in \partial^{\infty}(H)
$$

and Theorem 3.6 yields the result.

The nonhomogeneous case (assumption (iii) below) is more complicated. To start with we have

Proposition 4.2. Let $H$ be a $\sigma$-stable subalgebra of $L$ and set $D=\mathbb{D}_{L}(H)$. Fix a special complementary basis for $L \backslash D$ and assume that all the $\eta$ 's below are straightened monomials in that basis. For each of these finitely many distinct $\eta$, let $\gamma_{\eta} \in U(L)$. Assume that

(iii) $\operatorname{dim}_{K} H_{1}=\infty$.

If

$$
\sum_{\eta} \eta x \gamma_{\eta}=0 \quad \text { for all } x \in\left(H^{\prime}\right)^{\infty}
$$

where $H^{\prime}$ is a graded subspace of $H$ of finite codimension, then $\gamma_{\eta}=0$, for all $\eta$.

Proof. Since $\mathbb{D}_{L}(H)=\mathbb{D}_{L}\left(H^{\prime}\right)$, we can assume that $H=H^{\prime}$. Next write $\gamma_{\eta}=$ $\gamma_{\eta}^{\prime}+\gamma_{n}^{\prime \prime}$ as a sum of homogeneous terms with $\eta$ and $\gamma_{\eta}^{\prime}$ having the same parity and $\eta$ and $\gamma_{\eta}^{\prime \prime}$ having opposite parity. Then, for all homogeneous $x \in H^{\infty}$, we have

$$
0=\sum_{\eta} \eta x\left(\gamma_{\eta}^{\prime}+\gamma_{\eta}^{\prime \prime}\right)
$$

and taking homogeneous components yields

$$
\sum_{\eta} \eta x \gamma_{\eta}^{\prime}=0=\sum_{\eta} \eta x \gamma_{\eta}^{\prime \prime}
$$

Since these hold for all $x \in H^{\infty}$, we can therefore assume that each $\gamma_{\eta}$ is homogeneous with $\varepsilon(\eta)+\varepsilon\left(\gamma_{\eta}\right)=a$, where $a$ denotes a fixed parity. Throughout the proof, the parity $a$ will change as we modify the $\gamma$ 's. Suppose, by way of contradiction, that $\gamma_{\lambda} \neq 0$. Say $\varepsilon(\lambda)=e$ and set $N=\max _{\eta}\left\{\operatorname{deg} \eta, \operatorname{deg} \gamma_{\eta}\right\}$. We may assume that all $\gamma_{\eta}$ are nonzero, by deleting zero terms.

Let $h \in H_{1}$, and suppose $\varepsilon\left(\gamma_{\lambda}\right)=b$; then, for all $x \in H^{\infty}$ we have

$$
\sum_{\eta} \eta x h \gamma_{\eta}=0=\sum_{\eta} \eta x \gamma_{\eta} h
$$

so

$$
0=\sum_{\eta} \eta x\left(h \gamma_{\eta}+(-1)^{b} \gamma_{\eta} h\right)
$$

We now consider the expressions $h \gamma_{\eta}+(-1)^{b} \gamma_{\eta} h$. Suppose first that $\varepsilon(\eta)=$ $\varepsilon(\lambda)+1=e+1$. Then $\varepsilon\left(\gamma_{\eta}\right)=b+1$ and since $h \in H_{1}$,

$$
h \gamma_{\eta}+(-1)^{b} \gamma_{\eta} h=h \gamma_{\eta}-(-1)^{b+1} \gamma_{\eta} h=\gamma_{\eta}^{h} \text {, }
$$

and hence

$$
\operatorname{deg}\left(h \gamma_{\eta}+(-1)^{b} \gamma_{\eta} h\right)=\operatorname{deg} \gamma_{\eta}^{h} \leq \operatorname{deg} \gamma_{\eta} \leq N .
$$

On the other hand, if $\varepsilon(\eta)=\varepsilon(\lambda)$, then $\varepsilon\left(\gamma_{\eta}\right)=b$ and

$$
h \gamma_{\eta}+(-1)^{b} \gamma_{\eta} h=h \gamma_{\eta}-(-1)^{b} \gamma_{\eta} h+2(-1)^{b} \gamma_{\eta} h=\gamma_{\eta}^{h}+2(-1)^{b} \gamma_{\eta} h
$$


Again, $\operatorname{deg} \gamma_{\eta}^{h} \leq \operatorname{deg} \gamma_{\eta}$, but since $H_{1}$ is infinite dimensional, we can assume that $h$ is chosen outside of the space spanned by the supports of all such $\gamma_{\eta}$. In this case, $\operatorname{deg} \gamma_{\eta} h=\operatorname{deg} \gamma_{\eta}+1$ and thus $\operatorname{deg}\left(h \gamma_{\eta}+(-1)^{b} \gamma_{\eta} h\right)=\operatorname{deg} \gamma_{\eta}+1$. In other words, this process allows us to not increase the degree of all $\gamma_{\eta}$ with $\varepsilon(\eta)=e+1$ and to increase by 1 the degree of all $\gamma_{\eta}$ with $\varepsilon(\eta)=e$. Note also that

$$
\varepsilon\left(h \gamma_{\eta}+(-1)^{b} \gamma_{\eta} h\right)=\varepsilon\left(\gamma_{\eta}\right)+1
$$

so that this new identity

$$
0=\sum_{\eta} \eta x\left(h \gamma_{\eta}+(-1)^{b} \gamma_{\eta} h\right)
$$

has the property that, for all $\eta, \varepsilon(\eta)+\varepsilon\left(h \gamma_{\eta}+(-1)^{b} \gamma_{\eta} h\right)$ is constant. We can therefore continue this process and in a finite number of steps, we will obtain an identity

$$
\sum_{\eta} \eta x \tilde{\gamma}_{\eta}=0 \quad \text { for all } x \in H^{\infty}
$$

satisfying $\operatorname{deg} \eta \leq N, \operatorname{deg} \tilde{\gamma}_{\eta} \leq N$ if $\varepsilon(\eta)=e+1$, and $\operatorname{deg} \tilde{\gamma}_{\eta} \geq 3 N+1$ if $\varepsilon(\eta)=e$. In particular, $\operatorname{deg} \tilde{\gamma}_{\lambda} \geq 3 N+1$.

Now we start working on the left factors of $(*)$ as in the proof of Theorem 4.1. Let $x \in H^{\infty}$ and let $\tilde{h}$ be a homogeneous element of $H$. Since $\tilde{h} x \in H^{\infty}$, we have

$$
\sum_{\eta} \eta \tilde{h} x \tilde{\gamma}_{\eta}=0=\sum_{\eta} \tilde{h} \eta x \tilde{\gamma}_{\eta}
$$

and hence

$$
\sum_{\eta}\left(\tilde{h} \eta-(-1)^{\varepsilon(\tilde{h}) e} \eta \tilde{h}\right) x \tilde{\gamma}_{\eta}=0 .
$$

We consider the left-hand factors. If $\varepsilon(\eta)=e$ then $\tilde{h} \eta-(-1)^{\ell(\tilde{h}) e} \eta \tilde{h}=\eta^{\tilde{h}}$, while if $\varepsilon(\eta)=e+1$ then at least

$$
\operatorname{deg}\left(\tilde{h} \eta-(-1)^{\varepsilon(\tilde{h}) e} \eta \tilde{h}\right) \leq \operatorname{deg} \eta+1 \leq N+1 .
$$

Continuing this process at most $N$ steps, we see that for all $\delta \in \partial^{N}(H)$ we have

$$
\sum_{\varepsilon(\eta)=e} \eta^{\delta} x \tilde{\gamma}_{\eta}+\sum_{\varepsilon(\eta)=e+1} \eta(\delta) x \tilde{\gamma}_{\eta}=0 \quad \text { for all } x \in H^{\infty},
$$

where $\operatorname{deg} \eta(\delta) \leq N+N=2 N$. In particular, this holds for $x=1$ and recall that if $\varepsilon(\eta)=e+1$, then $\operatorname{deg} \tilde{\gamma}_{\eta} \leq N$. Thus the right-hand sum has degree at most $3 N$ and we conclude that

$$
\operatorname{deg} \sum_{\varepsilon(\eta)=e} \eta^{\delta} \tilde{\gamma}_{\eta} \leq 3 N \quad \text { for all } \delta \in \partial^{N}(H) .
$$

However, $\operatorname{deg} \eta \leq N$ for all $\eta$, so we can apply Proposition 3.5 with $r=N$ and $s=3 N$. We conclude, for all $\eta$ with $\varepsilon(\eta)=e$, that

$$
\operatorname{deg} \eta+\operatorname{deg} \tilde{\gamma}_{\eta} \leq s=3 N .
$$

But $\varepsilon(\lambda)=e$ and $\operatorname{deg} \tilde{\gamma}_{\lambda} \geq 3 N+1$, so we have a contradiction, thereby proving the result.

We can now combine the preceding two results. The weakness here is the assumption on $D$ in the case where $H_{1}$ is infinite dimensional. 
Theorem 4.3. Let $H$ be a graded subspace of $L$ and let $\alpha_{i}, \beta_{i} \in U(L)$ such that

$$
\alpha_{1} x \beta_{1}+\alpha_{2} x \beta_{2}+\cdots+\alpha_{v} x \beta_{v}=0 \quad \text { for all } x \in\left(H^{\prime}\right)^{\infty},
$$

where $H^{\prime}$ is a graded subspace of $H$ of finite codimension. Write each $\alpha_{i}=$ $\sum_{\eta} \eta \alpha_{\eta, i}$ specially based on $D=\mathbb{D}_{L}(H)$.

Assume that if $\operatorname{dim}_{K} H_{1}=\infty$, then $D=D_{0}$ is even. Then, for all $\eta$, we have

$$
\alpha_{\eta, 1} \beta_{1}+\alpha_{\eta, 2} \beta_{2}+\cdots+\alpha_{\eta, v} \beta_{v}=0 .
$$

In particular, if some $\alpha_{i}$ is not zero, then $\left\{\beta_{1}, \beta_{2}, \ldots, \beta_{v}\right\}$ is left linearly dependent over $U(D)$.

Proof. If $\operatorname{dim}_{K} H_{1}<\infty$, this is just Theorem 4.1. Therefore we can assume that $\operatorname{dim}_{K} H_{1}=\infty$ and hence that $D=D_{0}$ is even. Since each $\alpha_{\eta, i} \in U(D)=$ $U\left(D_{0}\right)$, we see that each $\alpha_{\eta, i}$ is even and, by Lemma 2.4, it follows that there exists a graded subspace $H^{\prime \prime}$ of finite codimension in $H^{\prime}$ with $\left[H^{\prime \prime}, \alpha_{\eta, i}\right]=0$, for all $\eta, i$. Replacing $H^{\prime}$ by $H^{\prime} \cap H^{\prime \prime}$, without loss of generality we can assume that $\left[H^{\prime}, \alpha_{\eta}, i\right]=0$, for all $\eta, i$. In particular, using property (1) and the fact that all the $\alpha_{\eta, i}$ are even, it follows that if $h$ is any homogeneous element of $H^{\prime}$ then $h$ commutes with $\alpha_{\eta, i}$. Thus all of $H^{\prime}$ commutes with $\alpha_{\eta, i}$.

Finally, let $x \in\left(H^{\prime}\right)^{\infty}$; then $x$ commutes with all $\alpha_{\eta, i}$, so the linear identity becomes

$$
0=\sum_{\eta, i} \eta \alpha_{\eta, i} x \beta_{i}=\sum_{\eta, i} \eta x \alpha_{\eta, i} \beta_{i}=\sum_{\eta} \eta x \gamma_{\eta} \quad \text { for all } x \in\left(H^{\prime}\right)^{\infty},
$$

where we set $\gamma_{\eta}=\sum_{i} \alpha_{\eta, i} \beta_{i}$. Proposition 4.2 now implies, using $\operatorname{dim}_{K} H_{1}=$ $\infty$, that $\gamma_{\eta}=0$, for all $\eta$.

It is clear that the above argument works without the assumption on $D$ if we merely assume that all the $\alpha_{\eta, i}$ have the same parity.

\section{APPLICATIONS OF DERIVATION AND LINEAR IDENTITIES}

In this section we obtain several consequences of the results on derivation and linear identities.

Corollary 5.1. Let $H$ be a $\sigma$-stable subspace of $L$ and set $D=\mathbb{D}_{L}(H)$. Then $U(D)$ is precisely the subring of $U(L)$ consisting of almost constants under the action of $H$.

Proof. Let $A$ be the ring of almost constants. By Lemma 2.4 we have $U(D) \subseteq$ $A$. Conversely, let $\alpha \in A$ and observe that for some $\sigma$-stable subspace $H^{\prime}$ of finite codimension in $H$ we have $1^{\delta} \alpha=\alpha^{\delta} 1$ for all $\delta \in \partial^{\infty}\left(H^{\prime}\right)$. Note that the $1^{\delta}$ term is needed to handle $\delta=1$.

Write $\alpha=\sum \eta \alpha_{\eta}$ specially based on $D$. Then, by Theorem 3.6, $\eta \neq 1$ implies that $\alpha_{\eta}=0$. Thus $\alpha=1 \alpha_{1} \in U(D)$.

Similarly we can speak about the almost centralizer of $H$ in $U(L)$. By definition $\alpha$ is in this almost centralizer if and only if it commutes with some $\sigma$-stable subspace $H^{\prime}$ of finite codimension in $H$. 
Corollary 5.2. Let $H$ be a $\sigma$-stable subspace of $L$ and set $D=\mathbb{D}_{L}(H)$. Then the almost centralizer of $H$ in $U(L)$ is a graded subring of $U(D)$. Furthermore if $\operatorname{dim}_{K} H_{1}=\infty$, then the almost centralizer is contained in $U(D)_{0}$.

Proof. Since $H$ is $\sigma$-stable it is clear that its almost centralizer is a $\sigma$-stable subring of $U(L)$. Suppose that $\alpha$ is a homogeneous element of this almost centralizer. Then $\alpha$ centralizers $H^{\prime}$, a subspace of $H$ of finite codimension, so $\alpha$ satisfies

$$
1 x \alpha=\alpha x 1 \text { for all } x \in\left(H^{\prime}\right)^{\infty} .
$$

Suppose first that either $\alpha$ is even or $\operatorname{dim}_{K} H_{1}<\infty$. Write $\alpha=\sum \eta \alpha_{\eta}$ specially based on $D$. Then, by Theorem 4.1 , we have $1 \alpha_{\eta}=0$ for all $\eta \neq 1$. Thus $\alpha=\alpha_{1} \in U(D)$.

Finally suppose by way of contradiction that $\alpha \neq 0$ is odd and $\operatorname{dim}_{K} H_{1}=$ $\infty$. If $h \in\left(H^{\prime}\right)_{1}$, then $\alpha^{h}=\alpha h+h \alpha=2 \alpha h$, and hence $\operatorname{deg} 2 \alpha h=\operatorname{deg} \alpha^{h} \leq$ $\operatorname{deg} \alpha$. On the other hand, since $\operatorname{dim}_{K} H_{1}=\infty$, we have choose $h$ not in the space spanned by the support of $\alpha$. But for this $h, \operatorname{deg} 2 \alpha h=1+\operatorname{deg} \alpha$ and we have the desired contradiction.

Suppose that $L_{0}=0$ and $L_{1}=K e_{1}+K e_{2}+\cdots+K e_{n}$ with $n$ odd. Then $U(L)$ is a Grassmann algebra and $\alpha=e_{1} e_{2} \cdots e_{n}$ is an odd central element. Thus we see that $\operatorname{dim}_{K} H_{1}=\infty$ is needed in the above.

We now move on, as in [BP], to study the semiprimeness and primeness of $U(L)$ and its relation to $U(\Delta(L))$. However the weakness of Theorems 4.1 and 4.3 leads to a less than perfect result. Indeed, for the most part, we can only consider graded primeness.

For the remainder of this section we fix a special complementary basis $B$ for $\mathbb{D}_{L}(L)=\Delta(L)$. Then every element $\alpha \in U(L)$ can be written uniquely as $\alpha=\sum_{\eta} \eta \alpha_{\eta}$ where $\eta$ is a straightened monomial in $B$ and where each $\alpha_{\eta} \in U(\Delta(L))$. If $\alpha \neq 0$ and if $n=\max \left\{\operatorname{deg} \eta \mid \alpha_{\eta} \neq 0\right\}$ then the leading coefficients of $\alpha$ are all those $\alpha_{\nu} \neq 0$ with $\operatorname{deg} \nu \neq n$.

Lemma 5.3. Let $\alpha=\sum_{\eta} \eta \alpha_{\eta}$ be a nonzero element described as above and let $\alpha_{\nu}$ be one of its leading coefficients.

(i) If $\alpha$ is homogeneous, then so is $\alpha_{\nu}$.

(ii) If $\gamma \in U(\Delta(L))$, then either $\alpha_{\nu} \gamma=0$ or $\alpha_{\nu} \gamma$ is a leading coefficient of $\alpha \gamma$.

(iii) If $\gamma$ is a homogeneous element of $U(\Delta(L))$ then either $\gamma \alpha_{\nu}=0$ or $\gamma \alpha_{\nu}$ is a leading coefficient of $\pm \gamma \alpha$.

Proof. (i) Since $B$ consists of homogeneous elements, each $\eta$ is homogeneous. In particular, if $\alpha$ is homogeneous then each $\alpha_{\eta}$ is also.

(ii) This is clear since $\alpha \gamma=\sum_{\eta} \eta\left(\alpha_{\eta} \gamma\right)$.

(iii) Let $\eta \neq 1$ be a straightened monomial in $B$ and write $\eta=z \eta^{\prime}$ with $z$ being its first factor. Note that $z$ is a homogeneous element of $L$. If $x$ is a homogeneous element of $\Delta(L)$, then $x z-(-1)^{\varepsilon(x) \varepsilon(z)} z x=z^{x}$ and hence

$$
x \eta=(-1)^{\varepsilon(x) \varepsilon(z)} z\left(x \eta^{\prime}\right)+z^{x} \eta^{\prime} .
$$

But $z^{x} \in \Delta(L)$, since $x \in \Delta(L)$, and therefore it follows by induction on $\operatorname{deg} \eta$ that

This of course also holds for $\eta=1$.

$$
x \eta=(-1)^{\varepsilon(x) \varepsilon(\eta)} \eta x+\sum_{\operatorname{deg} \lambda<\operatorname{deg} \eta} \lambda \beta_{\lambda} .
$$


Now suppose $\beta$ is a product of homogeneous elements of $\Delta(L)$. Since $\alpha=$ $\sum_{\eta} \eta \alpha_{\eta}$, it follows from the above that the $B$-monomials in $\beta \alpha$ all have degree $\leq \operatorname{deg} \nu$. Furthermore, the coefficient of $\nu$ in $\beta \alpha$ is given by $(-1)^{\varepsilon(\beta) \varepsilon(\nu)} \beta \alpha_{\nu}$.

Finally, $\gamma$ is a $K$-linear sum of such $\beta$ 's and, since $\gamma$ is homogeneous, $\varepsilon(\beta)=\varepsilon(\gamma)$. Thus all $B$-monomials in $\gamma \alpha$ have degree $\leq \operatorname{deg} \nu$ and the $\nu$ coefficient is equal to $(-1)^{\varepsilon(\gamma) \varepsilon(\nu)} \gamma \alpha_{\nu}$. This clearly yields the result.

If $A$ is a $K$-subspace of $U(L)$, we let $\pi(A)$ be the linear span of the leading coefficients of all $0 \neq \alpha \in A$. We also say that $A$ is $L$-stable if $\alpha^{x} \in A$ for all $\alpha \in A$ and all homogeneous $x \in L$. Basic properties are as follows:

Lemma 5.4. Let $J \neq 0$ be a graded ideal of $U(L)$. Then $\pi(J)$ is a nonzero graded, L-stable ideal of $U(\Delta(L))$. Furthermore $\pi(J)=\pi\left(J_{0}\right)+\pi\left(J_{1}\right)$.

Proof. If $\alpha=\sum_{\eta} \eta \alpha_{\eta} \in J$, then $\alpha^{\sigma}=\sum_{\eta} \eta(-1)^{\varepsilon(\eta)} \alpha_{\eta}^{\sigma}$. Thus the coefficients of $\alpha$ are linear combinations of the corresponding coefficients of $\alpha+\alpha^{\sigma}$ and $\alpha-\alpha^{\sigma}$. Since $J$ is graded, $\alpha+\alpha^{\sigma} \in J_{0}$, and $\alpha-\alpha^{\sigma} \in J_{1}$, so it follows that $\pi(J)=\pi\left(J_{0}\right)+\pi\left(J_{1}\right)$. Lemma 5.3 (i) now implies that $\pi(J)$ is spanned by homogeneous elements and hence $\pi(J)$ is graded.

Since $\pi(J)$ is closed under addition, (ii) and (iii) of the preceding lemma imply that $\pi(J)$ is a nonzero ideal of $U(\Delta(L))$. It remains to show that $\pi(J)$ is $L$-stable. For this, let $x$ be a homogeneous element of $L$. Now, if $\beta$ is a homogeneous element of $J$, then $\beta^{x}=x \beta-(-1)^{\varepsilon(x) \varepsilon(\beta)} \beta x \in J$. Thus since $J$ is graded, it follows that $J^{x} \subseteq J$.

Let $\alpha=\sum_{\eta} \eta \alpha_{\eta}$ be any element of $J$ and let $\alpha_{\nu}$ be a leading coefficient with $\operatorname{deg} \nu=n$. We compute the leading coefficients of $\alpha^{x} \in J$. Since each $\eta$ is homogeneous, we have

$$
\alpha^{x}=\sum_{\eta}\left(\eta^{x} \alpha_{\eta} \pm \eta \alpha_{\eta}^{x}\right)
$$

Furthermore, note that $\operatorname{deg} \eta^{x} \leq \operatorname{deg} \eta \leq n$. Thus if we write each $\eta^{x}$ as $\eta^{x}=\sum_{\tau} \tau\left(\eta^{x}\right)_{\tau}$ then $\operatorname{deg} \tau+\operatorname{deg}\left(\eta^{x}\right)_{\tau} \leq \operatorname{deg} \eta$. It follows from all of this that all $B$-monomials in $\alpha^{x}$ have degree $\leq n$ and that the coefficient of $\nu$ is $\pm \alpha_{\nu}^{x}$ plus a $K$-linear combination of leading coefficients of $\alpha$. But this coefficient and all leading coefficients of $\alpha$ are contained in $\pi(J)$. Thus $\alpha_{\nu}^{x} \in \pi(J)$ and $\pi(J)$ is $L$-stable.

We now prove

Theorem 5.5. If $A$ and $B$ are graded ideals of $U(L)$ with $A B=0$, then $\pi(A) \pi(B)=0$.

Proof. We first show that $\pi(A) B=0$ and, in view of Lemma 5.4, it suffices to show that $\pi\left(A_{a}\right) B=0$ for $a=0,1$. Let $\alpha=\sum_{\eta} \eta \alpha_{\eta}$ be a homogeneous element of $A$ and let $\beta \in B$. If $x \in L^{\infty}=U(L)$, then $\alpha x \beta \in A B=0$. Thus, since $\alpha$ is homogeneous, Theorem 4.1 implies that $\alpha_{\eta} \beta=0$ for all $\eta$. In particular, the leading coefficients of $\alpha$ annihilate $B$ and hence $\pi(A) B=0$.

Finally, recall that $\pi(A)$ is graded and let $\gamma$ be a homogeneous element of $\pi(A) \subseteq U(\Delta(L))$. If $\beta=\sum_{\eta} \eta \beta_{\eta} \in B$ has leading coefficient $\beta_{\nu}$ then, by Lemma 5.3(iii), either $\gamma \beta_{\nu}=0$ or $\gamma \beta_{\nu}$ is a leading coefficient of $\pm \gamma \beta$. But $\gamma \beta \in \pi(A) B=0$, so we must have $\gamma \beta_{\nu}=0$ and it follows that $\pi(A) \pi(B)=0$. 
We say that $U(\Delta(L))$ is graded $L$-prime if the product of any two nonzero graded, $L$-stable ideals of $U(\Delta(L))$ is nonzero and we say $U(L)$ is graded prime if the product of any two nonzero graded ideals of $U(L)$ is nonzero. Moreover, $U(\Delta(L))$ is $L$-semiprime if the square of any nonzero $L$-stable ideal is nonzero. We observe that if $A$ is a nilpotent ideal of either $U(L)$ or $U(\Delta(L))$ then $A+A^{\sigma}$ is a $\sigma$-stable nilpotent ideal and if $A$ is $L$-stable then so is $A+A^{\sigma}$ by (4).

Corollary 5.6. (i) $U(L)$ is graded prime if and only if $U(\Delta(L)$ ) is graded $L$ prime.

(ii) $U(L)$ is semiprime if and only if $U(\Delta(L))$ is L-semiprime.

Proof. Suppose $A$ is a graded, $L$-stable subspace of $U(L)$. If $\alpha \in A_{a}$ and $x \in L_{c}$ then $\alpha^{x}=x \alpha-(-1)^{a c} \alpha x$. Hence $L A \subseteq A L+A$ and $A L \subseteq L A+A$. It easily follows that $A U(L)=U(L) A$.

Now suppose $I, J$ are nonzero graded, $L$-stable ideals of $U(\Delta(L))$ such that $I J=0$. By the previous arguments, $I U(L)$ and $J U(L)$ are nonzero graded ideals of $U(L)$ such that $(I U(L))(J U(L))=(I J) U(L)=0$. As a result, if $U(L)$ is graded prime then $U(\Delta(L))$ is graded $L$-prime.

Conversely, if $I^{\prime}, J^{\prime}$ are nonzero graded ideals of $U(L)$ with $I^{\prime} J^{\prime}=0$, then by Theorem 5.5, $\pi\left(I^{\prime}\right)$ and $\pi\left(J^{\prime}\right)$ are nonzero graded, $L$-stable ideals of $U(\Delta(L))$ with $\pi\left(I^{\prime}\right) \pi\left(J^{\prime}\right)=0$. Thus, if $U(\Delta(L))$ is graded $L$-prime then $U(L)$ is graded prime and the graded prime case is settled.

The semiprime case follows by letting $I=J$ and $I^{\prime}=J^{\prime}$ in the above arguments and using our observation that $U(L)$ and $U(\Delta(L))$ contain nilpotent ideals if and only if they contain $\sigma$-stable nilpotent ideals.

It would be of interest to determine if $U(L)$ can be graded prime without being prime. Although Theorem 4.1 forces us to deal with graded primeness instead of primeness, we can use Theorem 4.3 to say more in the special case $\Delta(L)=0$.

Corollary 5.7. If $\Delta(L)=0$, then $U(L)$ is prime.

Proof. Assume that $\alpha U(L) \beta=0$ with $\alpha \neq 0$. Then for all $x \in L^{\infty}$ we have $\alpha x \beta=0$. Write $\alpha=\sum \eta \alpha_{\eta}$ specially based on $\Delta(L)$ and note that $\Delta(L)=\Delta(L)_{0}=0$. Thus by Theorem 4.3, $\alpha_{\eta} \beta=0$, for all $\eta$. But $\alpha \neq 0$ so $\alpha_{\eta} \neq 0$, for some $\eta$. Since $\alpha_{\eta} \in U(\Delta(L))=K$, we conclude that $\beta=0$.

Since $U(L)$ is prime when $\Delta(L)=0, U(L)$ has a symmetric Martindale ring of quotients $Q=Q_{S}(U(L))$. We know that any automorphism of $U(L)$ extends to one of $Q$. Thus $\sigma$ extends to an automorphism of $Q$ of order 2 . This implies that $[$,$] extends to Q$ and we study the action of $L$ on $Q$.

Corollary 5.8. Let $\Delta(L)=0$ and set $Q=Q_{S}(U(L))$. Then the almost constants in $Q$ under $L$ and the almost centralizer of $L$ in $Q$ are both equal to $K$. In particular, $U(L)$ is centrally closed.

Proof. Since $U(L)$ is prime, if $A \triangleleft U(L)$ with $A \neq 0$, then $A \cap A^{\sigma} \neq 0$. Thus every nonzero ideal contains a $\sigma$-stable nonzero ideal.

It is clear that the almost constants and almost central elements of $Q$ are $\sigma$-stable sets. Thus it suffices to consider homogeneous elements of those sets. Let $q$ be a homogeneous element of $Q$. By definition and the above, there exist nonzero $\sigma$-stable ideals $A, \tilde{A}$ of $U(L)$ with $A q \subseteq U(L)$ and $q \tilde{A} \subseteq U(L)$. 
Fix $0 \neq \alpha \in A$ with $\alpha$ homogeneous and set $\alpha q=\beta \in U(L)$. Let $\tilde{\alpha}$ be an arbitrary element of $\tilde{A}$ and set $q \tilde{\alpha}=\tilde{\beta}$.

Suppose first that $q$ is an almost constant, so that $q^{x}=0$ for all homogeneous $x \in L^{\prime}$, with $L^{\prime}$ a subspace of $L$ of finite codimension. If $\delta \in \partial^{\infty}\left(L^{\prime}\right)$, then $\beta^{\delta}=(\alpha q)^{\delta}=\alpha^{\delta} q$, by Lemma 1.1 (even when $\delta=1$ ), so

$$
\beta^{\delta} \tilde{\alpha}=\alpha^{\delta} q \tilde{\alpha}=\alpha^{\delta} \tilde{\beta} \quad \text { for all } \delta \in \partial^{\infty}\left(L^{\prime}\right) .
$$

It follows from Theorem 3.6, since $\Delta(L)=0$ and $U(\Delta(L))=K$, that there exist $a, b \in K$, not both zero, with $a \tilde{\alpha}=b \tilde{\beta}$. Furthermore $a$ and $b$ depend only on $\alpha$ and $\beta$ and hence are independent of $\tilde{\alpha}$. Finally $a \tilde{\alpha}=b \tilde{\beta}=b q \tilde{\alpha}$, so $(a-b q) \tilde{A}=0$ and hence $a-b q=0$. If $b=0$, then $a=0$, a contradiction. Thus $b \neq 0$ and $q=a b^{-1} \in K$.

Now let $q$ be almost central so that $q$ commutes with a subspace $L^{\prime}$ of finite codimension in $L$. It follows that for all $x \in\left(L^{\prime}\right)^{\infty}$ we have $\beta x=\alpha q x=\alpha x q$, so

$$
\beta x \tilde{\alpha}=\alpha x q \tilde{\alpha}=\alpha x \tilde{\beta} \quad \text { for all } x \in\left(L^{\prime}\right)^{\infty} .
$$

Since $\Delta(L)=0$ is even, Theorem 4.3 yields $a \tilde{\alpha}=b \tilde{\beta}$, with $a, b \in K$ depending only on $\alpha, \beta$. Furthermore not both $a$ and $b$ are zero. As above this yields $q=a b^{-1} \in K$.

We close this paper with the superalgebra version of [BP, Corollary 6.6].

Theorem 5.9. $U(L)$ is Artinian if and only if it is finite dimensional. In particular, this occurs if and only if $L$ is finite dimensional with $L_{0}=0$ when char $K=0$.

Proof. By Jacobson's Theorem, if char $K=p>0$ then $U(L)$ is finite dimensional if and only if $\operatorname{dim}_{K} L<\infty$. Similarly, by the Poincaré-BirkhoffWitt Theorem, if char $K=0$ then $U(L)$ is finite dimensional if and only if $\operatorname{dim}_{K} L<\infty$ and $L_{0}=0$. When $U(L)$ is finite dimensional it is certainly Artinian and our concern is with the converse.

If $I$ is a right ideal of $U\left(L_{0}\right)$, then $I U(L)$ is a right ideal of $U(L)$ with $I U(L) \cap U\left(L_{0}\right)=I$. Thus any chain of right ideals of $U\left(L_{0}\right)$ induces a corresponding chain in $U(L)$ and we conclude that $U\left(L_{0}\right)$ is also Artinian. Therefore, if char $K=0$, it is clear that $L_{0}=0$ and, if char $K=p$, then by Corollary 6.6 of [BP], $L_{0}$ is finite dimensional. Thus, in both cases, it suffices to show that $L_{1}$ is finite dimensional.

Let $A$ be a finite-dimensional subspace of $L_{1}$ and let $B$ be the $K$-linear span of all $a^{\delta}$ with $a \in A$ and $\delta \in \partial^{\infty}\left(L_{0}\right) . B$ is clearly an $L_{0}$-stable subspace of $L_{1}$ and, since $U\left(L_{0}\right)$ is finite dimensional, $B$ is also finite dimensional. Thus, if $L_{1}$ is infinite dimensional, there exists a chain $B_{(1)} \subset B_{(2)} \subset \cdots$ of $L_{0}$-stable finite-dimensional subspaces of $L_{1}$. Therefore $L_{0} \oplus B_{(1)} \subset L_{0} \oplus B_{(2)} \subset \ldots$ is a strictly ascending chain of finite-dimensional supersubalgebras of $L$, which are restricted if char $K=p$. Furthermore they yield $\left(L_{0} \oplus B_{(1)}\right) U(L)$ $\subset\left(L_{0} \oplus B_{(2)}\right) U(L) \subset \cdots$ a strictly ascending chain of right ideals of $U(L)$. However, this contradicts the fact that $U(L)$ is also Noetherian and thus we conclude that $L_{1}$ is finite dimensional. 


\section{REFERENCES}

[BP] J. Bergen and D. S. Passman, Delta methods in enveloping rings, J. Algebra 133 (1990), 277-312.

[B] E. J. Behr, Enveloping algebras of Lie superalgebras, Pacific J. Math 130 (1987), 9-25.

[S] M. Scheunert, The theory of Lie superalgebras, Lecture Notes in Math., vol. 716, SpringerVerlag, Berlin, 1979.

[P] V. M. Petrogradskij, Identities in the enveloping algebra for modular Lie superalgebras, J. Algebra 145 (1992), 1-21.

Department of Mathematics, DePaul University, Chicago, Illinois 60614

E-mail address: matjnb@depaul.bitnet

Department of Mathematics, University of Wisconsin, Madison, Wisconsin 53706

E-mail address: passman@math.wisc.edu 